\title{
Amplitude equations for pattern forming systems
}

\author{
M. van Hecke \\ Lorentz Institute, Leiden University, \\ P.O. Box 9506, 2300 RA Leiden, the Netherlands \\ P. C. Hohenberg \\ AT\&T Bell Laboratories \\ Murray Hill NJ 07974 USA. \\ and \\ W. van Saarloos \\ Institute Lorentz, University of Leiden, \\ P.O. Box 9506, 2300 RA Leiden, the Netherlands
}

\begin{abstract}
In this paper, based on the lectures by $\mathrm{PCH}$ and $\mathrm{WvS}$ at this summer school, we give an introduction to amplitude equations which describe slow modulations in space and time of patterns occuring in systems driven out of equilibrium. Emphasis is on general ideas rather than detailed formalism. The first part introduces the phenomenology of the well-known Rayleigh-Bénard instability and the basic linear stability and bifurcation theory used to describe the development of patterns. In the second part we derive the amplitude equations that govern the time evolution of patterns and discuss simple solutions of these equations. In the third part we consider some physical systems displaying patterns and their amplitude equations. In the fourth and final part we discuss the physical interpretation of more complicated solutions of the amplitude equations and compare the theory with numerical simulations and experiments.
\end{abstract}

This article appeared in Fundamental Problems in Statistical Mechanics VIII, H. van Beijeren and M. H. Ernst, eds (North-Holland, Amsterdam, 1994).

NB! Keep in mind that the article is somewhat dated by now; we make it available as there are not too many introductory articles on the subject. A more extended version of this article appeared in the thesis of Martin van Hecke in 1996. If you would like to have a copy of this thesis, write to mvhecke@lorentz.leidenuniv.nl 


\section{Contents}

1 Patterns. 4

1.1 Introduction. . . . . . . . . . . . . . . . . . 4

1.2 The Rayleigh-Bénard instability. . . . . . . . . . . . . . . . 6

1.3 Linear analysis. . . . . . . . . . . . . . . . . . . . . . . . . . . . . . . . . . . . .

1.4 Instabilities. . . . . . . . . . . . . . . . . . . . . 11

1.4.1 Bifurcations. . . . . . . . . . . . . . 11

1.4.2 Convective and absolute instabilities. . . . . . . . . . 14

1.4.3 Gradient dynamics. . . . . . . . . . . . . . . . . 14

2 Amplitude equations. $\quad 16$

2.1 Derivation of amplitude equations. . . . . . . . . . . 16

2.2 Comparison between the RGL and CGL equations. . . . . . . 22

2.3 Phase winding solutions and secondary instabilities. . . . . . . 23

2.4 Two-dimensional amplitude equations. . . . . . . . . . . 26

2.5 Noise. . . . . . . . . . . . . . . . . . 28

3 Physical examples. $\quad 29$

3.1 Rayleigh-Bénard convection revisited. . . . . . . . . . . . 29

3.1.1 Busse balloon. . . . . . . . . . . . . . . . 30

3.1.2 Convection in binary fluids. . . . . . . . . . . . . 31

3.1.3 Electrohydrodynamic convection. . . . . . . . . 32

3.2 Taylor-Couette flow. . . . . . . . . . . . . . . . . . . . 33

3.3 Parametric surface waves. . . . . . . . . . . . . . 34

3.4 Directional solidification. . . . . . . . . . . . . . . 34

3.5 Thermal noise. . . . . . . . . . . . . . . 36

3.6 Noise sustained structures. . . . . . . . . . . . . . . . 37

4 Beyond the phase winding solutions. $\quad 39$

4.1 The Eckhaus instability . . . . . . . . . . . . . . . . . . 40

4.2 Topological defects . . . . . . . . . . . . . . . . . . . . . . . . . . . . . . . 41

4.3 The Benjamin-Feir instability . . . . . . . . . . . . 42

4.3.1 Spatio-temporal chaos. . . . . . . . . . . . 43

4.4 One-dimensional coherent structures. . . . . . . . . . . . . . . 44

4.4.1 Sources and sinks. . . . . . . . . . . . . . 45

4.4.2 Pulses ..................... 47 
4.4 .3 Fronts ........................ . . 48

5 Concluding remarks. $\quad 48$

$6 \quad$ Suggested further reading $\quad 49$

$\begin{array}{ll}\text { References } & 49\end{array}$ 


\section{Patterns.}

\subsection{Introduction.}

In this paper we present a general theoretical description of the dynamics of nonequilibrium patterns close to the threshold of the instability that leads to their formation. As explained in more detail below, such nonequilibrium patterns occur e.g. in convection, in crystal growth and in reaction-diffusion systems such as oscillatory chemical reactions.

In Fig. 1 snapshots are shown of crystal growth in a system with a constant temperature gradient, known as directional solidification. This particular experiment shows the growth of a nematic phase into the isotropic phase of a liquid crystal. Each photo is taken at a different value of the growth velocity. As the picture illustrates, for values of the growth velocity smaller than some critical value $v_{c}$, the growing interface (the curve in each picture) remains straight (topmost picture), while above $v_{c}$ (about $2.5 \mu \mathrm{m} / \mathrm{s}$ in this experiment), the interface develops spatial modulations. The formation of these periodic patterns is due to a finite wavelength instability at $v_{c}$. Note that the modulation is weak close to $v_{c}$, and that the strength of the modulation increases with increasing $v-v_{c}$. The weakly nonlinear behaviour of this growth pattern close to the instability threshold is an example of the type of pattern formation we wish to discuss here.

The starting point for the theoretical analysis consists of equations of motion of the physical system displaying pattern forming behaviour. These are often a deterministic set of nonlinear partial differential equations:

$$
\partial_{t} U(x, t)=G\left[U, \partial_{x} U, \ldots ; R\right]
$$

where $U$ is the order parameter such as the height of the interface and $G$ is in general a nonlinear function of $U$ and its spatial derivatives and of $R$, which is a control parameter like $\left(v-v_{c}\right)$ in our example. These equations sometimes need to be supplemented by stochastic terms describing noise, but for a large macroscopic pattern forming system the noise is rather small and can often be neglected as we will discuss later in section 2.5. A typical class of equations of interest are so called reaction-diffusion equations of the form

$$
\partial_{t} U=D \nabla^{2} U+f(U, R)
$$

which for $f \equiv 0$ is the diffusion equation with well known behaviour ( $U$ is in general an $n$-component vector). A nonzero $f$ models reaction in the 

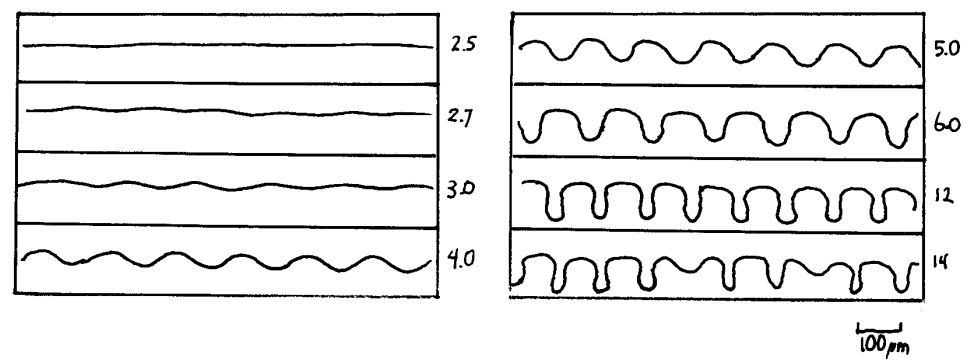

Figure 1: Directional solidification experiment, in which an isotropic liquid grows with constant velocity (indicated by the numbers on each panel) in a constant temperature gradient and freezes into a nematic phase. For increasing velocities (down), the solidification front displays a transition to a spatially periodic interface pattern, in which nonlinearities become increasingly important as the velocity is increased. After [1].

system, and the behaviour of the solutions of this equation can then be highly complex. The aim of the theory is to describe the solutions which are likely to be reached starting from physical initial conditions and to persist for long times.

In general the nonlinear equations cannot be solved analytically, and one therefore aims to describe their solutions qualitatively or perturbatively. It will turn out that patterns typically emerge after a control parameter exceeds a certain critical value and that often the amplitude of the pattern grows continuously from zero when the control parameter is increased beyond its critical value. One then first constructs solutions of the linearized equations of motion, which are exact in the limit that the control parameter goes to its critical value. Then one takes into account the nonlinearities that start to play a role for nonzero amplitude, that is for control parameter above its critical value, by means of perturbation theory. The perturbation of the linear pattern is governed by the amplitude equations. In other words: $A$ large number of pattern forming phenomena can be analyzed perturbatively by using so-called amplitude equations, which describe slow modulations in space and time of a simple basic pattern that can be determined from the linear analysis of the equations of motion of the physical system.

The form of the amplitude equation depends only on the nature of the 
linear instability, but not on other details of the system. The most important distinction is whether the basic pattern is stationary, leading to the real amplitude equation, or intrinsically time dependent, in which case an equation with complex coefficients describes the amplitude. As noted before the form of these equations is independent of details of the underlying system, only the coefficients in the amplitude equations reflect the physical details. The description in terms of amplitude equations therefore can be used to understand something of the universal pattern forming behaviour displayed by a number of different physical systems.

One should realize that the amplitude equations are only strictly valid for weakly nonlinear conditions, i.e. close to threshold. In stronger nonlinear regimes these equations can at most provide qualitative information. Within their range of validity, however, the amplitude equations yield an almost ${ }^{1}$ complete description of the effects that are crucial in pattern formation outside of equilibrium.

We will proceed by introducing some pattern forming systems and by elucidating some of the methods used to analyze their patterns. Recently some review papers ${ }^{2}$ have appeared both on the amplitude equation approach and on general aspects of nonequilibrium pattern formation. We will therefore confine ourselves to introducing and illustrating the main ideas of the approach and refer to these reviews for further discussion.

\subsection{The Rayleigh-Bénard instability.}

Probably the most famous pattern-forming system is the Rayleigh-Bénard experiment, where a horizontal layer of fluid is heated from below. Since the hot fluid expands, the vertical temperature gradient across the fluid results in a density gradient. Therefore, we encounter a destabilizing force, the buoyancy force, since the colder, heavier fluid would like to fall down so as to minimize the gravitational energy. The viscosity of the fluid has a stabilizing effect, and for small temperature gradients the fluid remains at rest and there is only heat conduction in the system. Small perturbations of the stationary conducting state decay so the conducting state is lineary stable.

However, when the temperature gradient exceeds a certain critical value,

\footnotetext{
${ }^{1}$ Exceptions are nonadiabatic effects such as those discussed by Bensimon et.al. [2].

${ }^{2}$ See section 6 .
} 
the viscosity can no longer balance the buoyancy force and the conductive state becomes unstable. It is useful to define the so called Rayleigh number $R$, which measures the ratio of destabilizing buoyancy force to the viscous force $(\mathrm{CH} \text { II A })^{3}$, and is the important control parameter of the system. So when $R$ increases above a certain critical value $R_{c}$, small perturbations of the basic state grow and a so-called convecting pattern emerges. Since not all the cold fluid can fall down simultaneously, the fluid will start to move up and down in a certain pattern, and the onset of convection therefore breaks the homogeneity of the basic conducting state. The simplest ordering of such a convective pattern consists of parallel rolls, as shown in Fig. 2.

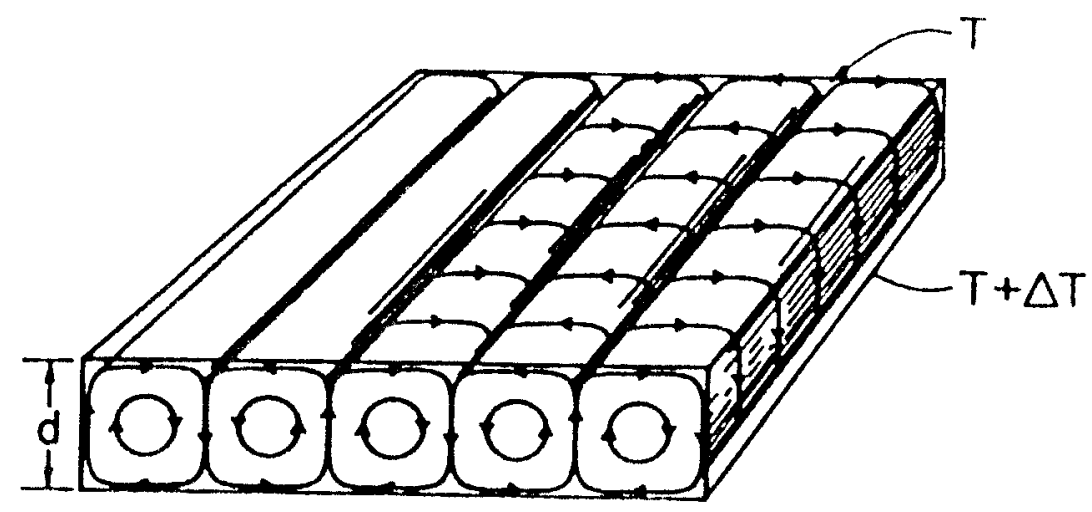

Figure 2: Sketch of parallel convecting rolls in a Rayleigh-Bénard experiment.

The situation sketched above is the simplest way in which patterns are formed in physical systems. By injecting energy into a system, typically a homogeneous equilibrium state becomes unstable above a certain threshold; as a result of this instability space-time patterns emerge above this threshold. To get an idea of the questions which one faces in pattern formation, let us take a look at the top view of a Rayleigh-Bénard experiment in a cylindrical cell.

A number of features of the patterns are visible and some questions immediately arise.

\footnotetext{
${ }^{3}$ References to a specific section $\mathrm{x}$ of the review paper by Cross and Hohenberg listed in section 6 are denoted as $\mathrm{CH} \mathrm{x}$.
} 


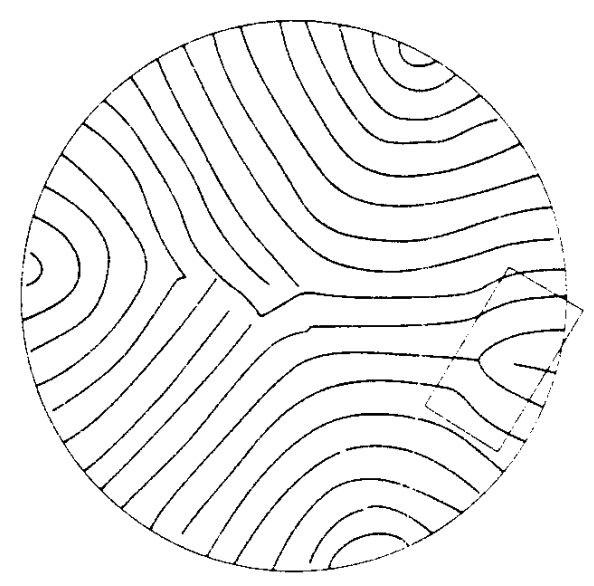

Figure 3: Top view of a roll pattern in a Rayleigh-Bénard experiment. The curves indicate the location of the centers of the convecting rolls. After [3].

- In large areas of the cell the patterns consist of almost equally spaced, parallel rolls like in Fig. 2. The wave vector that describes this periodicity varies only slowly throughout the cell. Why is this and how can we understand it?

- The pattern itself may in general evolve slowly in time; how can one describe this motion?

- In a few places rolls split, merge or end. These so called defects play an important role in the dynamics of the pattern.

- Due to the rotational symmetry of the system in the plane, there is no preferential direction of the rolls. However, the rolls apparently try to align themselves perpendicular to the sidewall, and this has important consequences for the final pattern.

- If the control parameter is increased far above its critical value, it is possible that the pattern itself becomes unstable against so called secondary instabilities. If the control parameter is increased even further, 
the pattern may become chaotic or turbulent, i.e. disordered in space and time.

In this paper we will not try to discuss all the points raised above, but will instead focus on the basic theoretical ingredients for describing the dynamics of the pattern close to threshold. As we will show, in large portions of the cell, the pattern can be considered as a slow modulation in space and time of a simple basic structure, in this case parallel rolls. Therefore, the fluid motion is described as the product of a slowly varying amplitude and an underlying pattern with faster dependence in space and/or time. This approach leads to a separation of space-time scales, and to the determination of an equation of motion for the amplitude that describes the slow evolution of the pattern.

But before we derive the amplitude equation valid in the weakly nonlinear regime (i.e. $R$ near $R_{c}$ ), we first focus on the linear stability analysis and the different bifurcations associated with changes in stability.

\subsection{Linear analysis.}

Although the fluid is not at rest in the convecting state of the RayleighBénard experiment in a stationary layer of fluid, the regular basic pattern that emerges is time independent. In different systems, like for instance Rayleigh-Bénard convection in a rotating layer of fluid, the basic pattern may be explicitly time dependent and may consist of traveling waves. The principle of linear analysis is not different in this case, but for the sake of simplicity we will limit the discussion here to time independent basic patterns. When the system is rotationally invariant in the plane, the direction of the wave vector is immaterial, and we can for simplicity take the wavevector parallel to the $x$-direction. In the present section we shall neglect all $y$-variation, i.e. we consider one-dimensional patterns. We take the system infinitely long in the $x$-direction, so as to avoid studying the effects of lateral boundaries, and moreover we assume left-right reflection symmetry $(x \rightarrow-x)$. In this case we may look for Fourier-mode-like solutions for the linearized equations of motion of the form

$$
U(x, y, z, t)=F[z] e^{\sigma t+i q x}+c . c .,
$$

where c.c. denotes the complex conjugate, $U$ is a vector describing the physical fields such as velocity and temperature and $F[z]$ is some basic mode in 
the vertical direction whose details are not of interest to us now; the growth rate $\sigma$ is a real number for the present case and $q$ is the wave vector of the mode. Substituting the ansatz (3) into the linearized equations of motion of the fluid, we find the dispersion relation $\sigma(q)$. This dependence of the growth rate on $q$ is sketched for three values of the reduced control parameter $\varepsilon=\left(R-R_{c}\right) / R_{c}$ in Fig. 4 .

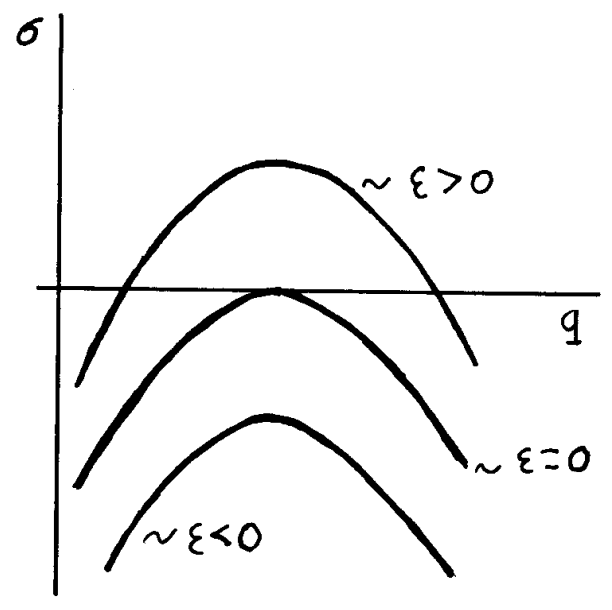

Figure 4: Growth rate $\sigma$ as a function of the wave number for various $\varepsilon$.

The homogeneous basic state is stable if there is no $q$ such that the growth rate is positive, and this is clearly the case for $\varepsilon<0$. If the control parameter passes through zero, there starts to emerge a small band of wave numbers around $q_{c}$ that correspond to growing modes, therefore the homogeneous state becomes unstable and a pattern with wave numbers inside the band around $q_{c}$ emerges.

Since the fluid equations are relatively complicated, it is useful to introduce a simple model equation which illustrates the linear instability sketched above. The linear part follows immediately from Fig. 4 and the $x \rightarrow-x$ symmetry, so we find an equation of the form $\partial_{t} u=\varepsilon u-D\left(\partial_{x}^{2}+q_{c}^{2}\right)^{2} u+$ nonlinear terms. If the ansatz $u(x, t)=e^{\sigma t+i q x}+$ c.c. is substituted into this equation, we find that $\sigma=\varepsilon-D\left(q_{c}^{2}-q^{2}\right)^{2}$, so indeed the growth rate behaves as sketched in Fig. 4. 
Let us require that this model equation be invariant under a change of sign of $u$, then the simplest nonlinearity is a cubic one. If we include a cubic term of the form $-\alpha u^{3}(\alpha>0)$, we find the so-called Swift-Hohenberg equation [6]:

$$
\partial_{t} u=\varepsilon u-\left(\partial_{x}^{2}+q_{c}^{2}\right)^{2} u-u^{3} .
$$

Here we have used the fact that with a proper rescaling of space and $u, \alpha$ and $D$ can be put equal to one. Note that this equation is much simpler than the fluid equations to be discussed below. For instance $u$ is meant to be a single real function of $x$ and $t$, whereas in the equations of motion for the Rayleigh-Bénard experiment we have a vector velocity and temperature field depending on $x, y, z$ and $t$.

Now that we have a simple model to describe the instability of the homogeneous state in a one-dimensional Rayleigh-Bénard experiment, we can ask ourselves whether this model also describes the birth of the convecting state. In the next section we will show that when the homogeneous $u=0$ state becomes unstable, a new periodic state emerges which describes the periodic pattern found in the Rayleigh-Bénard experiment.

\subsection{Instabilities.}

It should be noted that since one in general cannot define a free energy for pattern forming systems and since there is usually no thermodynamic limit involved, the transition to convection is not a phase transition, but is associated with a qualitative change in the behaviour of solutions of a set of equations, which occurs when a control parameter is varied. This is called a bifurcation. Above threshold, i.e. for $\varepsilon>0$, it turns out that generally there exists a continuous family of solutions, e.g. periodic roll patterns with wavelengths in a band. This is another difference between the bifurcations found in pattern forming systems and phase transitions, where the transition is between two thermodynamically different states.

\subsubsection{Bifurcations.}

We will proceed by analyzing the bifurcation that occurs for the SwiftHohenberg model when $\varepsilon$ passes through zero. From the linear stability 
analysis we know that the fastest growing mode has wave number $q_{c}$, and so we attempt to find a solution of the Swift-Hohenberg equation of the form

$$
u(x, t) \sim \cos \left(q_{c} x\right)+h \cdot h .
$$

where $h . h$. denote higher harmonics which we need to include because the nontrivial solutions of the Swift-Hohenberg equation cannot be written down in closed analytic form.

If we substitute Eq. (5) into the full (including nonlinearity) SwiftHohenberg equation (4), it is found that for small $\varepsilon$ we can construct a solution of the form

$$
u(x, t)=\sqrt{\frac{4 \varepsilon}{3}} \cos \left(q_{c} x+\phi\right)+\mathcal{O}\left(\varepsilon^{3 / 2}\right) \cos \left(3 q_{c} x\right)+\mathcal{O}\left(\varepsilon^{3 / 2}\right) \cos \left(q_{c} x\right),
$$

with $\phi$ arbitrary. It can be shown that this solution is stable for small $\varepsilon$. We will interpret this periodic state as the analogue of the convecting state in the Rayleigh-Bénard experiment. If we now sketch the amplitude of the "conducting" $u=0$ and "convecting" $u \sim \cos \left(q_{c} x\right)$ solutions as a function of the control parameter and denote the linear stability of these solutions by continuous (stable) and dashed (unstable) curves, we obtain a so called bifurcation diagram.

We now focus on the types of bifurcations that are important in the study of patterns. In Fig. 5 we sketch the bifurcation diagrams of a supercritical (forward) pitchfork bifurcation as found in the Swift-Hohenberg equation and in the Rayleigh-Bénard experiment, and of a subcritical (backward) pitchfork bifurcation as found for instance in the Rayleigh-Bénard experiment in binary fluid mixtures (see sec. 3.1.2).

In the supercritical case, when we increase $\varepsilon$ through zero, we find that the homogeneous $u=0$ state becomes lineary unstable at $\varepsilon=0$ and a new solution bifurcates from the $u=0$ state. For $\varepsilon>0$ the system will be pushed from the unstable "conducting" state (by infinitesimally small perturbations such as noise) and end up in this "convecting" $u \neq 0$ state. Note that for this supercritical bifurcation the amplitude of the "convecting" pattern grows like a square root above onset, and when we let the control parameter pass through zero, the amplitude of the stable and therefore experimentally observed mode varies continuously. In that sense the supercritical bifurcation is reminiscent of a second order phase transition. 

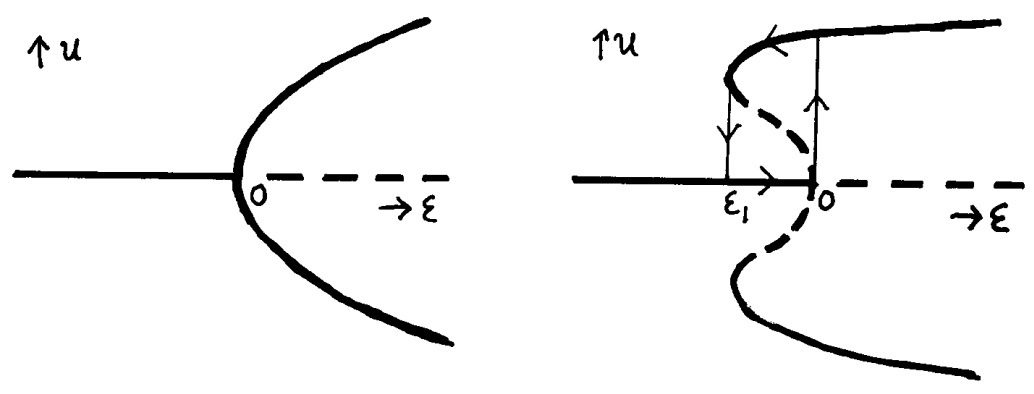

Figure 5: Supercritical and subcrical pitchfork bifurcations. Solif lines refer to stable solutions, dashed lines to unstable ones (see CH II A 2).

The subcritical bifurcation diagram, on the other hand, describes a different situation. If $\varepsilon$ is increased through zero, the $u=0$ state loses stability and the system will make a jump and will end up on some $u \neq 0$ branch. If we now decrease $\varepsilon$ again, the system will remain on this branch untill $\varepsilon<\varepsilon_{1}$ and then jump back to the $u=0$ state. This hysteresis and discontinous change of the amplitude is similar to a first order phase transition. Subcritical bifurcations occur for instance in Rayleigh-Bénard experiments in binary fluid mixtures, and considerably complicate the analysis, because one in general cannot make an expansion for small $\varepsilon$ and $u$, as can and will be done below for the supercritical case.

Having introduced the distinction between supercritical and subcritical bifurcations, we will add another concept; a bifurcation is called stationary if the resulting basic pattern is time independent as in the Swift-Hohenberg equation, and it is called oscillatory (also known as Hopf bifurcation) if the critical mode is time-dependent. For oscillatory instabilities with nonzero wave vector the basic pattern is a traveling wave mode of the form $\exp [i(q x-$ $\omega t)]$. Note that the distinction between these two types is independent of the bifurcation being sub- or supercritical. Although as mentioned above the fluid is in motion in the Rayleigh-Bénard experiment, the basic roll pattern is stationary just above threshold, and the onset of convection occurs via a stationary supercritical (pitchfork) bifurcation. We will later see examples of equations and physical systems with Hopf bifurcations. 


\subsubsection{Convective and absolute instabilities.}

The question of linear stability or instability is slightly more complicated if there is a mean flow in the system. Suppose we are looking at the flow of a liquid through a pipe. The simple homogeneous state then corresponds to laminar flow. Now suppose that this flow becomes unstable, because we increase the velocity of the fluid. Thus, small perturbations of the laminar state will grow in time. However, when perturbations are advected away by the overal fluid velocity faster than they grow, then at a fixed position the perturbations will eventually die out, as shown in Fig. 6a. In this case the instability is called convective. When perturbations grow faster than they are advected away, or more precisely, if there exists a position in the lab frame such that some infinitesimal perturbations do not decay, then the instability is called absolute (Fig. 6b). Note that the definition of convective and absolute instability is frame-dependent. A system undergoing a Hopf bifurcation to travelling waves is convectively unstable in the lab frame immediately above threshold (CH VI C 1).

CONVECTIVE INSTABILITY

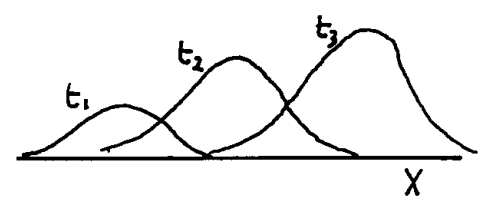

(a)
ABSOLUTE INSTABILITY

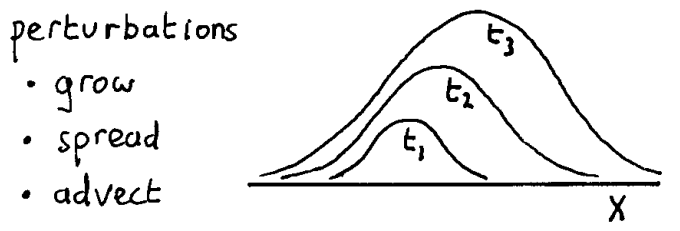

(b)

Figure 6: Convective and absolute instabilities.

\subsubsection{Gradient dynamics.}

Although for pattern forming systems one cannot in general define a free energy whose minima determine the solution at long times, for the SwiftHohenberg equation there exists a so called Lyapunov function $F$ which behaves like a free energy: this $F$ will not increase during the time evolution of 
the equation, and the dynamics tends to drive the solutions toward a local minimum of $F$. To be more specific, the Swift-Hohenberg equation (4) can be written as

$$
\partial_{t} u=-\frac{\delta F}{\delta u}
$$

where

$$
F[u]:=\frac{1}{2} \int d x\left\{\left[\left(\partial_{x}^{2}+q_{c}^{2}\right) u\right]^{2}-\varepsilon u^{2}+\frac{1}{2} u^{4}\right\} .
$$

From this it follows that the dynamics decreases $F$, because

$$
\frac{d F}{d t}=\int d x \frac{\delta F}{\delta u} \frac{\partial u}{\partial t}=-\int d x\left(\frac{\delta F}{\delta u}\right)^{2} \leq 0 .
$$

Therefore the dynamics is "downhill" and is sometimes called gradient dynamics. Note that the precise time evolution of $u$ is not easily determined from the Lyapunov function, but its importance is that final states can be obtained by finding minima of $F$. We should stress again that this sort of gradient dynamics is the exception, not the rule.

Now that we have explained how one can understand the birth of patterns via instabilities, we proceed in the next section to derive the so called amplitude equations which describe these patterns near threshold. 


\section{Amplitude equations.}

\subsection{Derivation of amplitude equations.}

In this section we will explicitly demonstrate how an amplitude equation is derived from a given set of starting equations showing a finite wavelength instability. To fix the ideas, one could think of Rayleigh-Bénard convection restricted to patterns of parallel rolls, therefore only allowing for modulations in one direction, so that the amplitude equation is one-dimensional. In section 2.4, we shall briefly discuss the extension to the two-dimensional case. We will indicate how such a derivation is performed, but to minimize technical details we will only illustrate the explicit calculation for the aforementioned Swift-Hohenberg equation (4). The principles of the derivation of amplitude equations are the same for many types of pattern forming systems. The method consists of an expansion of the solution $U$ of the full equations of motion in the control parameter $\varepsilon$, writing the leading term of this expansion as the product of a slowly varying amplitude and a basic pattern which is the critical solution of the linearized equations of motion; in the Rayleigh-Bénard case this basic pattern consists of parallel rolls of wavenumber $q_{c}$. The goal is to derive an equation of motion for the slowly varying amplitude.

We will start our derivation by showing how from the linear stability analysis the slow scales can be obtained. Therefore, return to Fig. 4 showing the linear growth rate $\sigma$ as a function of the wavenumber $q$ for three values of the control parameter $\varepsilon$. For small $\varepsilon$, only wavenumbers close to $q_{c}$ are important, and one finds to lowest order in $\varepsilon$ and $\left(q-q_{c}\right)$ that

$$
\sigma=\left(\frac{\partial \sigma}{\partial \varepsilon}\right)_{0} \varepsilon+\frac{1}{2}\left(\frac{\partial^{2} \sigma}{\partial q^{2}}\right)_{q_{c}}\left(q-q_{c}\right)^{2}+\ldots,
$$

so, according to linear theory, only modes with wave vectors in a band around $q_{c}$ of width $\approx \sqrt{\varepsilon}$ are growing. These are the modes which play the dominant role in the long time behaviour of the full nonlinear equations of motion. The following trivial observation explains why this long time behaviour can be described by slow modulations of the critical mode, at least in the bulk of the system: If a solution of the full equations of motion contains a mode with $x$-dependence $e^{i q x}$, we can write this as $e^{i\left(q-q_{c}\right) x} e^{i q_{c} x}$, and since all dominant modes have $\left|q-q_{c}\right| \leq \varepsilon^{1 / 2}$, the $x$-dependence of the full solution can indeed be approximated as the product of a slow spatial modulation $e^{i\left(q-q_{c}\right) x}$ and 
the critical mode $e^{i q_{c} x}$. When for these dominant modes the phase factor $\left(q-q_{c}\right) x$ changes by an amount of order unity, the spatial scale on which this happens goes like $1 /\left(q-q_{c}\right) \sim \varepsilon^{-1 / 2}$. We therefore expect the length scale of the modulations to scale like $\varepsilon^{-1 / 2}$.

The growth rate of the relevant modes varies lineary in $\varepsilon$, and from an analogous argument it is found that the characteristic time scale of the modulations is proportional to $\varepsilon^{-1}$. Finally, from the shape of the bifurcation curve (Fig. 5a) it follows that the amplitude grows as $\varepsilon^{1 / 2}$. We therefore expect solutions of the fully nonlinear equations to be of the form

$$
U=\varepsilon^{1 / 2} A(X, T) U_{\text {lin }}+\text { c.c. }+ \text { h.o.t. }
$$

where $X:=\varepsilon^{1 / 2} x$ and $T:=\varepsilon t$ are the explicit slow scales, $U_{\text {lin }}$ is the critical solution of the linearized equations of motion and h.o.t. denotes higher order terms. For the present discussion we confine ourselves to one spatial dimension. Our task is to find an equation for the amplitude $A$. As we shall see, the higher order terms arise naturally in this expansion as well, but their amplitude is driven by the amplitude $A$; this is called slaving.

To derive the amplitude equation, we construct a weakly nonlinear expansion of the full equations of motion, by assuming

$$
U=\varepsilon^{1 / 2} U_{0}+\varepsilon U_{1}+\varepsilon^{3 / 2} U_{2}+\ldots
$$

where it will turn out that the leading order term $U_{0}$ can be written as $A(X, T) U_{\text {lin }}$ as in (11). If we substitute this expansion into the equations of motion, we naturally arrive at a separation of fast and slow scales.

To see how this works in practice, let us now apply the scheme to the Swift-Hohenberg equation (4) in one dimension:

$$
\partial_{t} u=\varepsilon u-\left(\partial_{x}^{2}+q_{c}^{2}\right)^{2} u-u^{3}:=\varepsilon u-\mathcal{L} u-u^{3},
$$

by substituting the ansatz (12) into this equation. When derivatives are taken of products of the form (11) in the ansatz, the chain rule shows that we need to replace $\partial_{t}$ and $\partial_{x}$ as follows:

$$
\partial_{t} \rightarrow \varepsilon \partial_{T}, \partial_{x} \rightarrow \partial_{x}+\varepsilon^{1 / 2} \partial_{X}
$$

where in the expression for $\partial_{x}$, the $x$ on the LHS acts on spatial dependence on all length scales, and on the RHS $x$ only acts on the fast spatial dependence 
of the $e^{ \pm i q_{c} x}$ terms, while $\partial_{X}$ acts on the slow spatial variable. Carrying out this separation of scales we find for the linear operator $\mathcal{L}$

$$
\begin{aligned}
\mathcal{L}=\left(\partial_{x}^{2}+q_{c}^{2}\right)^{2} & \rightarrow(\underbrace{\partial_{x}^{2}+q_{c}^{2}}_{L}+2 \varepsilon^{1 / 2} \partial_{x} \partial_{X}+\varepsilon \partial_{X}^{2})^{2} \\
& =L^{2}+4 \varepsilon^{1 / 2} L \partial_{x} \partial_{X}+\varepsilon\left(2 L+4 \partial_{x}^{2}\right) \partial_{X}^{2}+\mathcal{O}\left(\varepsilon^{3 / 2}\right),
\end{aligned}
$$

where for notational convenience, $L$ is defined as $\partial_{x}^{2}+q_{c}^{2}$. If we now substitute this together with the ansatz for $u$ into the Swift-Hohenberg equation, we find

$$
\begin{aligned}
&\left\{L^{2}+\right.\left.4 \varepsilon^{1 / 2} L \partial_{x} \partial_{X}+\varepsilon\left(\partial_{T}-1+\left(2 L+4 \partial_{x}^{2}\right) \partial_{X}^{2}\right)+\mathcal{O}\left(\varepsilon^{3 / 2}\right)\right\} \times \\
&\left\{\left(\varepsilon^{1 / 2} u_{0}+\varepsilon u_{1}+\varepsilon^{3 / 2} u_{2}+\mathcal{O}\left(\varepsilon^{2}\right)\right\}+\varepsilon^{3 / 2} u_{0}^{3}+\mathcal{O}\left(\varepsilon^{2}\right)=0\right.
\end{aligned}
$$

When we collect orders in $\varepsilon$ this leads to a hierarchy of equations of which the lowest is

$$
\mathcal{O}\left(\varepsilon^{1 / 2}\right):\left(\partial_{x}^{2}+q_{c}^{2}\right)^{2} u_{0}=0
$$

so at leading order we find an equation whichdetermines the linearized solution

$$
u_{0}=e^{i q_{c} x} A_{0}(X, T)+e^{-i q_{c} x} A_{0}^{*}(X, T)
$$

where it should be noted that the complex amplitude function $A_{0}$ can be completely arbitrary at this level, since the linear operator $\left(\partial_{x}^{2}+q_{c}^{2}\right)^{2}$ only acts on the fast scales. At the next order we find

$$
\mathcal{O}(\varepsilon): \underbrace{4\left(\partial_{x}^{2}+q_{c}^{2}\right) \partial_{x} \partial_{X} u_{0}}_{=0}+\left(\partial_{x}^{2}+q_{c}^{2}\right)^{2} u_{1}=0,
$$

where the first term is zero because $L u_{0}=0$. Analogous to the previous order, it therefore follows that

$$
u_{1}=e^{i q_{c} x} A_{1}(X, T)+c . c .,
$$

where $A_{1}$ also can be chosen arbitrarily at this level. It can however be determined from higher equations in the hierarchy. The previous equation 
does not help us, but at the next order we will find an equation of motion for $A_{0}$, the leading slow amplitude. We find

$$
\mathcal{O}\left(\varepsilon^{3 / 2}\right): L^{2} u_{2}+\underbrace{4 L \partial_{x} \partial_{X} u_{1}}_{=0}+(\partial_{T}-1+\underbrace{2 L \partial_{X}^{2}}_{\rightarrow 0}+4 \partial_{x}^{2} \partial_{X}^{2}+u_{0}^{2}) u_{0}=0
$$

which by eliminating the zero terms and expanding the nonlinear term leads to

$$
L^{2} u_{2}+\left[e^{i q_{c} x}\left(\partial_{T}-1-4 q_{c}^{2} \partial_{X}^{2}+3\left|A_{0}\right|^{2}\right) A_{0}+\text { c.c. }\right]+\left(e^{3 i q_{c}} A_{0}^{3}+\text { c.c. }\right)=0 .(23
$$

From this it follows that $u_{2}$ is of the form $e^{i q_{c} x} A_{2}+e^{3 i q_{c} x} B_{2}+$ c.c., where $A_{2}$ and $B_{2}$ are slow amplitudes which are not determined at this level. However, the crucial point is the following: $L^{2} u_{2}$ does not contain any $e^{i q_{c} x}$ dependence, since $L A e^{i q_{c} x}=0$ for all slow amplitudes $A$. So, in order to satisfy Eq. (23), the coefficient of $e^{i q_{c} x}$ in (23) must vanish, i.e. $A_{0}$ must satisfy

$$
\partial_{T} A_{0}=A_{0}+4 q_{c}^{2} \partial_{X}^{2} A_{0}-3\left|A_{0}\right|^{2} A_{0}
$$

This is the amplitude equation we wanted to derive. By a simple scaling of $X$ and $A_{0}$ by a constant factor, we can eliminate the constants $4 q_{c}^{2}$ and 3. The ultimate justification for having chosen the scales of space, time and amplitude as $\varepsilon^{-1 / 2}, \varepsilon^{-1}$ and $\varepsilon^{1 / 2}$, respectively, follows from the self consistency of the above expansion. Indeed this form of the amplitude equation is independent of $\varepsilon$.

The calculation of amplitude equations for more complicated systems with a forward stationary bifurcation is technically more involved, but the principles are the same: by separating in the equations of motions all derivatives into a slow and a fast part (i.e. $\partial_{x} \rightarrow \partial_{x}+\varepsilon^{1 / 2} \partial_{X}$ ) and assuming for $U$ an expansion of the form (12), a systematic expansion of the equations of motion is obtained. The first equation in this hierarchy corresponds to the linearized equation of motion and therefore does not tell us anything about the amplitude $A_{0}$. The second equation is also of no help, but the third equation is in general of the form

$$
\mathcal{L}_{f} U_{2}=\operatorname{RHS}[A]
$$

where $\mathcal{L}_{f}$ is the fast part of the linear operator of the starting equation. The above relation has a solution if and only if the RHS is orthogonal to the zero 
space of the linear operator $\mathcal{L}_{f},{ }^{4}$ and this condition gives us the general amplitude equation:

$$
\tau_{0} \partial_{t} A=\xi_{0}^{2} \partial_{x}^{2} A+\varepsilon A-g_{0}|A|^{2} A,
$$

which now is written down for the fast scales $x$ and $t$.

Although the coefficients $\tau_{0}, \xi_{0}$ and $g_{0}$ can be calculated from the full equations describing the physical problem under study ${ }^{5}$, for convenience we can scale them out by a suitable choice of space, time and amplitude scales. Note that we cannot scale away the sign of $g_{0}$, because for positive $g_{0}$ the nonlinear term is stabilizing and we have a supercritical bifurcation, while a negative $g_{0}$ gives rise to a destabilizing effect on the amplitude and the bifurcation is subcritical; higher order stabilizing nonlinearities are then necessary to obtain a stationary solution. We now assume a supercritical bifurcation as in Rayleigh-Bénard convection, and after the aforementioned scaling we obtain

$$
\partial_{t} A=\partial_{x}^{2} A+\varepsilon A-|A|^{2} A
$$

We prefer to keep $\varepsilon$ explicit in (27), so as to avoid a control parameter dependent rescaling. This will make it easier to consider what happens when $\varepsilon$ goes through zero.

Equation (27) arises naturally near any stationary supercritical bifurcation when the system is translationally invariant and reflection symmetric $(x \rightarrow-x)$. The latter symmetry dictates that the second order term $\partial^{2} / \partial x^{2}$ arises as the lowest order spatial derivative, while the form of the cubic term is prescribed by the requirement that the equation be invariant upon multiplying $A$ by an arbitrary phase factor $\exp (i \phi)$ : this corresponds to translating the pattern by a distance $\phi / q_{c}$, so translational invariance implies that the equation for $A$ has to be invariant under $A \rightarrow A e^{i \phi}$.

Equation (27) has the form of the Ginzburg-Landau equation for superconductivity in the absence of a magnetic field and is often refered to as the Ginzburg-Landau model. To distinguish it from the amplitude equation for traveling waves given below, we will refer to it as the real Ginzburg-Landau equation (RGL), since the coefficients in this equation are real. Note that the

\footnotetext{
${ }^{4}$ According to the Fredholm theorem, discussed in appendix A of $\mathrm{CH}$.

${ }^{5}$ From the equation for the linear growth rate (10) it follows that $\tau_{0}^{-1}=\partial \sigma / \partial \varepsilon$ and $\xi_{0}^{2} \tau_{o}^{-1}=-(1 / 2) \partial^{2} \sigma / \partial q^{2}$.
} 
amplitude itself is a complex valued function in order to take proper account of the translational symmetry.

If the instability is to traveling waves, i.e. if the pattern which emerges is intrinsically time-dependent, the resulting amplitude equation generalizes to the complex Ginzburg-Landau equation (CGL). Still the principle of derivation is the same, but since the solutions of the linearized equations of motion are traveling waves of the form $e^{i\left(q_{c} x-\omega_{c} t\right)}$, where $\omega_{c}$ is the critical frequency, we write the lowest order solution as $U_{0}=e^{i\left(q_{c} x-\omega_{c} t\right)} A_{0}(X, T)+$ c.c. Since there is now both fast and slow time dependence, $\partial_{t}$ transforms to $\partial_{t}+\varepsilon \partial_{T}$. Performing the $\varepsilon$ expansion and scaling away superfluous constants, we find as amplitude equation the complex Ginzburg-Landau equation (CGL):

$$
\partial_{t} A+v_{g} \partial_{x} A=\left(1+i c_{1}\right) \partial_{x}^{2} A+\varepsilon A-\left(1-i c_{3}\right)|A|^{2} A
$$

where $c_{1}$ and $c_{3}$ are real coefficients, and $v_{g}$ is the group speed. Naively, one would expect $\left(1+i c_{0}\right)$ in front of the $\varepsilon A$ term, but unlike $c_{1}$ and $c_{3}, c_{0}$ can be transformed away by going to a rotating frame, i.e. setting $\tilde{A}=e^{-i c_{0} t} A$. Solutions of the CGL equation are qualitatively different for different values of $c_{1}$ and $c_{3}$. These coefficients can be calculated from the basic equations of motion by performing the same expansion as in the real case.

We have written only one CGL equation for a single amplitude, i.e. we have broken the symmetry under reflection $(x \rightarrow-x)$. If the starting system is itself symmetric both left- and right-moving traveling waves can exist. For such systems, one actually obtains two coupled CGL equations, one for the amplitude of the left-moving waves and one for the amplitude of the rightmoving waves. Depending on the nonlinear interaction terms, one can either have a situation in which standing waves are favoured, or one in which one wave suppresses the other in the bulk of the system. In the latter case, one can effectively use a single CGL equation like (28), at least in an infinite or periodic system, and the $v_{g} \partial_{x} A$ term can be eliminated by a Galilean transformation; we then end up with:

$$
\partial_{t} A=\left(1+i c_{1}\right) \partial_{x}^{2} A+\varepsilon A-\left(1-i c_{3}\right)|A|^{2} A,
$$

which is the form of the CGL equation we will use most often. In the limit $c_{1}, c_{3} \rightarrow 0$ we recover the RGL equation. 


\subsection{Comparison between the RGL and CGL equations.}

To derive both amplitude equations, the only essential assumption we used was that there is a supercritical bifurcation with wave number unequal to zero. If this is the case, the amplitude equations are generically the real or the complex one, depending on whether the bifurcation is stationary or oscillatory. Therefore, these equations describe the weak nonlinear regime of many physical systems, and are in a sense universal. For systems with subcritical bifurcations the situation is more complicated; if the amplitude of the bifurcating mode is small, one can still do perturbation theory and derive amplitude equations, but in general this is not sufficient and higher order terms must be added, so amplitude equations can, at most, give qualitative behaviour. When the instability occurs for $q_{c}=0$, then the form of the amplitude equations is different, but for supercritical bifurcations an amplitude description can still be given ( $\mathrm{CH}$ IV A 1).

At first glance the real and the complex equation look rather similar, but it turns out that the behaviour of solutions of these equations is very different.

It is easy to check that the RGL equation (27) can be written in the form

$$
\frac{\partial A}{\partial t}=-\frac{\delta \mathcal{F}}{\delta A^{*}}, \quad \text { with } \mathcal{F}=\int d x\left[\left|\frac{\partial A}{\partial x}\right|^{2}-\varepsilon|A|^{2}+\frac{1}{2}|A|^{4}\right],
$$

from which it follows that $d \mathcal{F} / d t \leq 0$. Thus, $\mathcal{F}$ plays the role of a 'free energy' or Lyapunov function, ${ }^{6}$ and many aspects of the dynamics of patterns can be simply understood in terms of the tendency of patterns to evolve towards the lowest free-energy state. In this sense, the dynamics of (27) is very thermodynamic-like and is called relaxational.

For $c_{1}, c_{3} \neq 0$, the CGL equation can not be derived from a Lyapunov function and it displays a much richer variety of dynamical behavior than the real equation (27). In fact, in the limit $c_{1}, c_{3} \rightarrow \infty$ the equation reduces to the Nonlinear Schrödinger equation, which is not only Hamiltonian but also integrable (it has the well-known soliton solutions). The fact that the CGL equation reduces to an equation possessing a Lyapunov function in one limit and to a Hamiltonian equation in another limit makes it very interesting from

\footnotetext{
${ }^{6}$ When $c_{1}=-c_{3}$, the CGL equation can, after going to a rotating frame setting $\tilde{A}=$ $e^{i c_{1} t} A$, be written as $\partial_{t} \tilde{A}=\left(1+i c_{1}\right)\left(\partial_{x}^{2} \tilde{A}+\varepsilon \tilde{A}-\left|\tilde{A}^{2}\right| \tilde{A}\right)=-\left(1+i c_{1}\right) \frac{\delta \mathcal{F}}{\delta \tilde{A}^{*}}$.
} 
a theoretical point of view. In addition, these two limits can be exploited as starting points for perturbation expansions.

\subsection{Phase winding solutions and secondary instabili- ties.}

We now proceed to discuss simple solutions of the real and complex GinzburgLandau equation. In the last chapter of these notes we will consider more complicated solutions, but in general these cannot be written down in closed, analytic form. To get a grip on these solutions, we first discuss the so-called phase winding solutions which can be found easily.

The RGL equation admits plane waves in space of the form $A=a e^{i q x}$, with $q^{2}=\varepsilon-a^{2}$. These phase winding solutions describe steady state periodic patterns with total wave number slightly bigger $(q>0)$ or slightly smaller $(q<0)$ than $q_{c}$.

For the CGL equation, there exists a band of traveling wave solutions $A=a e^{-i \omega t+i q x}$ with $\operatorname{Im}(\omega)=0$. Just as $q$ measures the difference between the wave number of the pattern and the critical wave number, $\omega$ measures the difference between the frequency of the pattern and the frequency of the critical mode, $\omega_{c}$. Note that the RGL equation does not permit these traveling wave solutions.

When we substitute the ansatz $A=a e^{-i \omega t+i q x}$ into Eq. (29), we obtain

$$
\omega=c_{1} q^{2}-c_{3} a^{2}, \quad q^{2}=\varepsilon-a^{2} .
$$

The expression for $\omega$ illustrates that $c_{1}$ is the coefficient which measures the strength of the linear dispersion, i.e. the dependence of the frequency of the waves on the wave number, while $c_{3}$ is a measure of the nonlinear dispersion.

So, the RGL equation admits spatially periodic solutions $e^{i q x}$ with wave vector $-\varepsilon^{\frac{1}{2}}<q<\varepsilon^{\frac{1}{2}}$, and the CGL equation admits travelling waves $e^{-i \omega t+i q x}$ with condition (31). What is the stability of these solutions? The linear stability analysis is quite straightforward but especially for the CGL equation algebraically involved. The stability analysis consists of simply substituting a trial function which is the sum of the phase winding solution and a suitable periodic perturbation into the corresponding amplitude equation and linearizing the reponse to the small perturbation. This results in a growth rate for the perturbation, which determines whether the phase winding solution is lineary stable or unstable (CH IV A). 
(a)

$$
C_{2}=C_{3}=0
$$

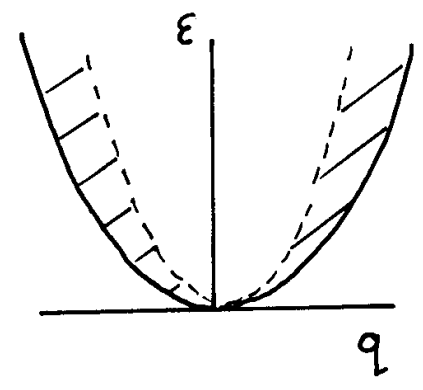

\section{$C_{1} C_{3}>1$}

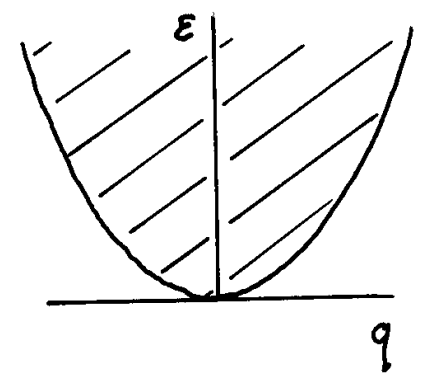

(b)

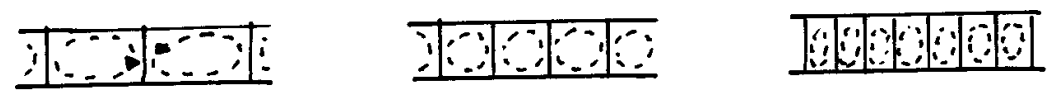

Figure 7: Illustration of the stability of phase winding solutions of the onedimensional real and complex Ginzburg-Landau equations. (a) The stability diagram for $c_{1}=c_{3}=0$ (left) and for $c_{1} c_{3}>1$ (right). (b) Sketch of three roll patterns in a Rayleigh-Bénard cell for $q$ close to the left edge of the band of allowed solutions, for $q \approx 0$, and for a $q$ close to the right edge of the band.

When a phase winding solution becomes unstable, this is a secondary instability since the solution itself emerged via an instability of the homogeneous $A=0$ state. These secondary instabilities tend to make the patterns more complex and account for some of the complexity found in real experiments, as we will see in sec. 3.1.1. For the real case the qualitative answer is well-known; consider the left part of Fig. 7a. For a given $\varepsilon>0$, the values of $q$ for which steady state solutions exist fall within the solid line. However, only the solutions within the dashed lines are stable - solutions corresponding to values of $q$ in the hatched region, close to the edge of the band, are linearly unstable. This instability is called the Eckhaus instability. Intuitively, one may understand it as follows. A wave number $q$ close to the left edge of the band corresponds to a smaller total wave number of the pattern, and hence a larger wavelength. When the wave vector is too small, as illustrated for a roll pattern in a Rayleigh-Bénard cell on the left of Fig. 7b, the pattern is unstable because a roll is so wide that it will split into three. Likewise, a $q$ near the right edge amounts to a pattern which is unstable because three 


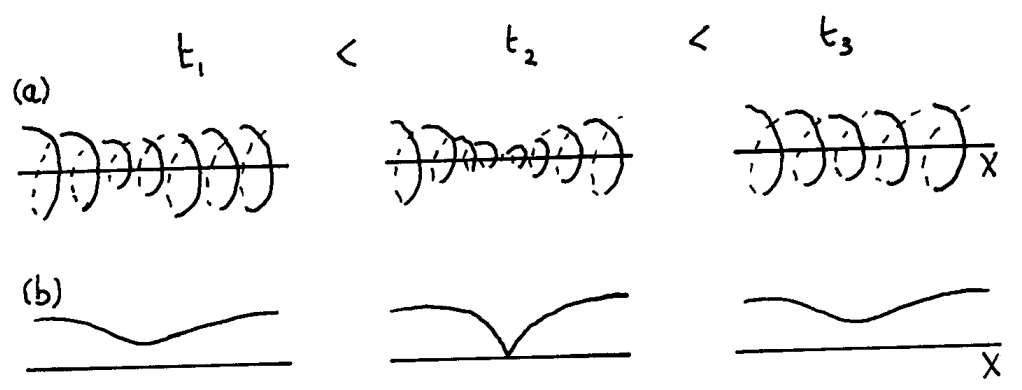

Figure 8: Illustration of the dynamical process by which phase winding solutions with too large $|q|$ go unstable. In (a) the complex envelope $A$ is plotted as a function of $x$ for three different times. Note that the plane perpendicular to the $x$-axis is a complex plane. In (b) the dynamics of $|A|$ is sketched; at time $t_{2}$ the phase slip occurs.

narrow rolls like those in the right part of Fig. 7b merge into one. Only patterns with wavelength close enough to the critical one (those in the center of Fig. $7 \mathrm{~b}$ with $q \approx 0$ ) are stable. Now in the Rayleigh-Bénard example of Fig. $7 \mathrm{~b}$ the phase difference of $A e^{i q_{c} x}$ between two points, divided by $2 \pi$, is equal to the number of pairs of rolls between these two points ${ }^{7}$. Thus when three rolls merge into one or when one roll splits into three, the number of phase windings of $A$ over a certain distance changes by one. But since the phase of $A$ is well defined and continuous whenever $|A|$ is nonzero, the only way the number of phase windings can change discontinuously in a localized region is if at some point in time and space $|A|=0$. At that point the phase is undefined, and so can "slip" by $2 \pi$. These points are called phase slip centers. Fig. 8 illustrates the rapid variation of the phase and the decrease in modulus $|A|$ which lead to such behavior.

For the CGL equation, the stability analysis is more complicated, since one has two free parameters $\left(c_{1}\right.$ and $\left.c_{3}\right)$ which can be adjusted. Just as in the Eckhaus instability it is found that within the range of possible phase winding solutions, a smaller band of these solutions is stable; if one leaves this band, one encounters the so-called Benjamin-Feir instability ${ }^{8}$, which corresponds

\footnotetext{
${ }^{7}$ The phase will be discussed in more detail in sec. 4.1 and 4.2.

${ }^{8}$ This instability is analogous to the Eckhaus instability of the RGL equation, but in
} 
to a wave becoming unstable by resonant excitation of sidebands. The size of this band is a complicated function of $c_{1}$ and $c_{3}$, but one important feature can be caught in a simple formula: If the so-called Newell criterion

$$
c_{1} c_{3}>1
$$

is valid, all phasewinding solutions are lineary unstable! This remarkable result leads to expectations that in this case chaos will occur, as is indeed observed in numerical simulations (see sec. 4.3.1).

\subsection{Two-dimensional amplitude equations.}

In a realistic Rayleigh-Bénard experiment as shown in Fig. 3 the pattern is explicitly two-dimensional so an extension of the amplitude equation considered above is needed to describe the pattern. We will show that the amplitude equation for isotropic (i.e. rotationally invariant) systems is anisotropic, whereas for anisotropic systems the corresponding amplitude equation can take an isotropic form. The reason for this somewhat paradoxical situation is that a periodic roll pattern breaks the rotational symmetry in an isotropic system, so transverse and longitudinal variations are qualitatively different, whereas in the anisotropic system they can be made the same by a simple scale change.

The term in the amplitude equation which is different from the onedimensional case is the spatial derivative term, which we can find by looking at the linear growth rate of plane waves. Suppose we have an isotropic system and that the basic pattern has a wavevector $\vec{q}_{0}$ in the $x$-direction. Then the growth rate $\sigma$ of modes with wavenumber $\vec{q}=\overrightarrow{q_{0}}+\vec{k}$ is given by a generalization of Eq. (10) of the form

$$
\sigma(q)=\tau_{0}^{-1}\left(\varepsilon-\xi_{0}^{2}\left|\vec{q}-\vec{q}_{0}\right|^{2}\right) \approx \tau_{0}^{-1}\left(\varepsilon-\xi_{0}^{2}\left(k_{x}+k_{y}^{2} / 2 q_{0}\right)^{2}\right),
$$

where we have kept the lowest order terms in each of $k_{x}$ and $k_{y}$ in expanding $\left|\vec{q}-\overrightarrow{q_{0}}\right|^{2}$ for small $\vec{k}$. Note that the difference in scaling in the two directions reflects the inherent symmetry breaking of the instability, which was here chosen with wave vector in the $x$-direction. The amplitude equation is now found to have the form

$$
\tau_{o} \partial_{t} A=\varepsilon A+\xi_{0}^{2}\left(\partial_{x}-\left(i / 2 q_{0}\right) \partial_{y}^{2}\right)^{2} A-g_{0}|A|^{2} A,
$$

the context of the CGL equation it is usually referred to as the Benjamin-Feir instability. 


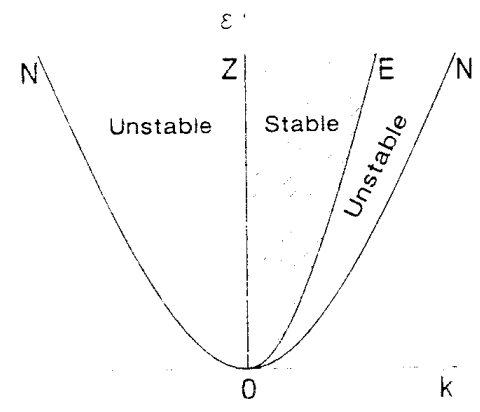

Figure 9: Stability balloon for states with wavevector $\vec{q}=\left(q_{0}+k\right) \hat{x}$ in the two-dimensional RGL equation. Within the curve $N$ phase winding solutions exist. $E$ and $Z$ denote the Eckhaus and zig-zag instabilities.

in the real case and its usual extension in the complex case. These equations correctly describe the variation of the pattern on a slow scale $\varepsilon^{1 / 2}$ parallel to the roll wave vector and $\varepsilon^{1 / 4}$ perpendicular to the wave vector. An important limitation of this amplitude equation therefore is that it only describes situations in which the rolls are almost everywhere parallel to a particular direction, here labeled the $x$-direction. The slow reorientation of the roll pattern over large angles commonly observed in experiments (see e.g. Fig. 3) cannot be accounted for by the present theory.

If the system is not invariant under rotations in the plane the amplitude equation takes the form

$$
\tau_{0} \partial_{t} A=\varepsilon A+\xi_{x}^{2} \partial_{x}^{2} A+\xi_{y}^{2} \partial_{y}^{2} A-g_{0}|A|^{2} A
$$

in the real case, and again the usual extension in the complex case. By a rescaling of the $x$ or $y$-coordinate one can bring the anisotropic equation into the isotropic form

$$
\tau_{0} \partial_{t} A=\varepsilon A+\xi^{2}\left(\partial_{x}^{2} A+\partial_{y}^{2} A\right)-g_{0}|A|^{2} A .
$$

These two-dimensional equations also have phase winding solutions, but the new feature is the occurence of the so-called "zig-zag" instability for negative values of $k_{x}$, which is a modulation of the phasewinding solutions parallel to the rolls, hence in the $y$-direction (Fig. 9). 


\subsection{Noise.}

In deriving the amplitude equations we assumed that the noise in the underlying physical system could be neglected. However, one should be careful with this. If the system is close to threshold, small stochastic terms have a potentially important effect. For instance, if the Rayleigh-Bénard system is just above threshold, perturbations of the conductive state grow and lead to the convective pattern. However, it is clear that the amplitude of these noisy perturbations determines how quickly a convecting state is reached, and without noise one in principle could remain in the unstable conducting state forever (see sec. 3.5). As a simple model to study the influence of noise, one can supplement the aforementioned amplitude equations by an additive Langevin (white) noise term. The prefactor of this term then has to be determined from a more detailed study of the underlying physical system. Although we will not go into detail on how to do this ${ }^{9}$, we note that at the very least any physical system is subjected to thermal fluctuations, and in sec. 3.6 we quote the strength of this thermal noise in various systems.

\footnotetext{
${ }^{9}$ The strength of the noise term in the amplitude equation can be determined by projecting the noise term in the original equations onto the slow mode. See sec. 3.5.
} 


\section{Physical examples.}

\subsection{Rayleigh-Bénard convection revisited.}

So far we have concentrated on the onset of convection in the RayleighBénard experiment for simple, isotropic liquids. The hydrodynamic equations describing this system are the Navier-Stokes equation supplemented with the heat equation and the mass conservation law. We are interested in the situation where a temperature difference is maintained between two horizontal plates. In general the parameters of the Navier Stokes equation like viscosity and thermal conductivity depend on temperature and density, and taking this coupling into account makes the equations very complicated. However, in the weakly nonlinear regime the temperature difference is typically of the order of $1 \mathrm{~K}$, so this coupling is not very important. The so-called "Boussinesq approximation" [4] only includes the temperature dependence in the all important buoyancy force term, and otherwise assumes an incompressible fluid with constant material parameters. In this approximation the fluid equations are:

$$
\begin{aligned}
\left(\partial_{t}+\vec{u} \cdot \vec{\nabla}\right) \vec{u} & =-\vec{\nabla}(P / \rho)+\nu \nabla^{2} \vec{u}-g \alpha T \hat{z}, \\
\left(\partial_{t}+\vec{u} \cdot \vec{\nabla}\right) T & =\kappa \nabla^{2} T, \\
\vec{\nabla} \cdot \vec{u} & =0
\end{aligned}
$$

where $g$ is the acceleration of gravity, $\nu$ is the viscous diffusivity, $\kappa$ is the thermal diffusivity, $\alpha$ is the thermal expansion coefficient, $\rho$ is the density and $\vec{u}, P$ and $T$ denote fluid velocity, pressure and temperature. These equations in principle should be supplemented by stochastic noise terms,

reflecting the small scale degrees of freedom of the molecular constituents of the fluid. These terms are however very small (as discussed in sec. 3.5, for ordinary Rayleigh-Bénard convection they typically turn out to be of relative order $10^{-9}$ ).

For the present discussion we shall neglect the stochastic forcing. To treat the onset of convection, we write down perturbation equations, perturbing around a state of steady conduction. We first rewrite the hydrodynamic equations in the Boussinesq approximation (37) - (39) in dimensionless units,

$$
\left(\frac{1}{\sigma} \partial_{t}-\nabla^{2}\right) \vec{u}+\vec{\nabla} P-\theta \hat{z}=-\frac{1}{\sigma}(\vec{u} \cdot \vec{\nabla}) \vec{u}
$$




$$
\begin{aligned}
\left(\partial_{t}-\nabla^{2}\right) \theta-R u_{z} & =-(\vec{u} \cdot \vec{\nabla}) \theta \\
\vec{\nabla} \cdot \vec{u} & =0
\end{aligned}
$$

where $\Delta T$ is the temperature difference between lower and upper plate, $R:=$ $g \alpha \Delta T d^{3} / \kappa \nu$ denotes the Rayleigh number, $\sigma:=\nu / \kappa$ is the Prandtl number which measures the ratio of thermal and viscous diffusivities $[\sigma$ should not be confused with the growth rate (10)] and $\theta$ is the deviation of the temperature from its equilibrium distribution. If $R$ is increased a pattern of parallel rolls emerges in simple cases. In a realistic experiment the horizontal layer of fluid extends in both $x$ and $y$ directions, so modulations close to onset can be described by the isotropic two-dimensional real amplitude equation (34).

We will proceed by describing what happens in more complicated situations. First we indicate what may happen if the Rayleigh number is not close to onset, and secondly we will describe convection in binary fluid mixtures and in the anisotropic case of a nematic liquid crystal.

\subsubsection{Busse balloon.}

We have seen that the simple phase winding solutions of the amplitude equations can become unstable against secondary instabilities, and an obvious extension is to the situation further away from threshold, as described by Eqs. (40) - (42). These equations are simple enough for the stability of the periodic roll structure to be described in detail. In a series of papers, Busse and coworkers [5] gave a complete classification of the secondary instabilities which occur in Rayleigh-Bénard convection in pure fluids. The simplest secondary instability is the Eckhaus instability, the well known modulation of the wave number of the pattern that is already found in the amplitude equation. Besides the Eckhaus instability a whole zoology of secondary instabilities is obtained, with exotic names like "zig-zag" (modulation of the pattern parallel to the rolls) and "skewed-varicose" (roughly a combination of zig-zag and Eckhaus). If we sketch the boundaries of the region in the space of wave number and control parameter where parallel rolls are stable, we obtain the so-called "Busse balloon" whose detailed form depends on Prandtl number only (Fig. 10). Inside this balloon the periodic roll structure is lineary stable, but if we cross one of its boundaries, the rolls are destabilized by a secondary instability. It is worth noting that only the Eckhaus and the

zig-zag instabilities are found in the amplitude equation, showing the limited 
range of validity of this equation. The other instabilities are only found if one includes higher order terms resulting from the full system (40) - (42). It is interesting to note the complexity of the destabilising scenarios which can occur in real fluids for higher Rayleigh numbers.

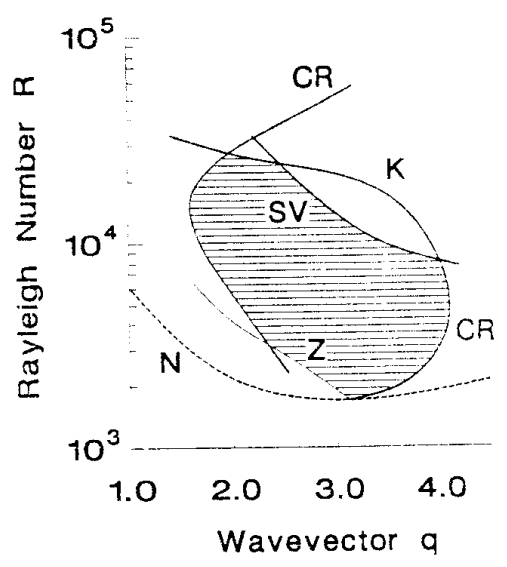

Figure 10: Busse balloon for $\sigma=7$, appropriate to water, where the different names for the various curves indicate the secondary instabilities that occur there. $N$ denotes the neutral curve, $E$ the Eckhaus instability, $Z$ stands for the zig-zag instability and $S V$ stands for the skewed-varicose instability. $K$ and $C R$ denote the "knot" and "cross roll" secondary instabilities, which are not discussed in the text. The Eckhaus instability is to the right of the cross-roll boundary in this case and is thus not shown. After [5].

\subsubsection{Convection in binary fluids.}

If one uses instead of a pure fluid a mixture of two fluids in the convection experiment, new phenomena appear. The important new feature is that apart from a concentration flow induced by ordinary diffusion, temperature differences also induce a concentration flow. The hydrodynamic equations (37) - (39) have to be supplemented by equations for the concentration $c$ of one of the components:

$$
\begin{aligned}
\partial_{t} c & =-\vec{\nabla} \cdot \vec{J}_{c}, \\
\vec{J}_{c} & =-D_{c}(\vec{\nabla} c+\psi \vec{\nabla} T)+c \vec{u},
\end{aligned}
$$


where $D_{c}$ is the diffusion coefficient of the component in question. The coupling between temperature and concentration flow is known as the Soret effect and the dimensionless coefficient which measures the strength of this effect, $\psi$, is called the separation ratio; $\psi$ can be both positive and negative, depending on the average concentration of the mixture. One can imagine that this effect is important in convection; if the sign of $\psi$ is such that the heavier fluid component flows to relatively hotter regions it has a stabilizing effect, whereas if the heavier component flows to colder regions it has a destabilizing effect.

Since the dynamics of the concentration is in general on a different time scale than the dynamics of fluid motion, the resulting behaviour can be quite rich. The ratio of time scales is given by the Lewis number $\mathcal{L}:=D_{c} / \kappa$ which is typically very small $\left(10^{-2}\right)$ for liquids. The most interesting point is that it turns out that for negative separation ratios (destabilizing), the onset of convection occurs via a Hopf bifurcation, so the roll pattern is time dependent, and consists of traveling waves. This is our first example of a physical system described by the CGL equation, and therefore RayleighBénard convection in binary fluids has been the object of intense study in the last few years.

There are two major problems, however, with the amplitude approach for this system. Due to the smallness of the Lewis number it turns out that an amplitude expansion can only be valid for very small amplitudes of the pattern. Moreover, the bifurcation turns out to be subcritical ( $g_{0}$ is negative), so small amplitudes are quite exceptional and in general a perturbative approach is not valid (CH IX A).

\subsubsection{Electrohydrodynamic convection.}

Nematics are liquids, usually formed of long anisotropic molecules with properties characterized by a single anisotropy axis, called the director. The dielectric constant for instance is coupled to this director and as a consequence electrical forces couple to the fluid flow. In addition the anisotropy of the conduction may be important, leading to charge build up, which in turn couples to electric fields. If a strong enough electrical field is applied across the nematic, electrohydrodynamic instabilities occur which produce roll patterns analogous to Rayleigh-Bénard convection. The destabilizing forces are in general much larger then the buoancy forces in ordinary convection systems, 
and consequently the size of the rolls is much smaller.

The typical geometry of an electrohydrodynamic convection experiment consists of a thin layer of nematic between two parallel plates separated by 10-100 $\mu \mathrm{m}$, across which a voltage is applied. The plates are treated to favor the alignment of the director in a particular orientation in the plane of the plates, so that the homogeneous quiescent state consists of the fluid with director pointing in one direction (say the $x$-direction) and no fluid flow. The instability develops when the voltage exceeds a certain critical value. The most familiar instability is a supercritical stationary bifurcation to a spatially periodic roll state normal to the $x$-direction; in this state there is fluid circulation coupled to the tilt of the director in the $x-z$ plane. Because of the anisotropy of the system, the amplitude equation can, by a constant scaling of the $x$ and $y$ directions parallel to the plates, be brought into the isotropic form (36).

The main advantages of electrohydrodynamic convection are the ability to control the flow by electromagnetic means and the small spatial scale of the convection rolls, which makes it possible to study systems with many rolls, so as to minimize the influence of lateral boundaries; in addition fluctuation effects can more easily be studied in such systems. The major disadvantage is that the electrohydrodynamic equations are very complicated.

\subsection{Taylor-Couette flow.}

This systems consists of two concentric rotating cylinders with fluid in between. If we rotate the cylinders the fluid also starts to rotate, and a pressure gradient emerges because of the centrifugal forces. We are then in a situation which is similar to the Rayleigh-Bénard experiment, and one again looks at the balance of destabilizing centrifugal forces and viscosity which acts as a stabilizing force. The dimensionless control parameter measuring this ratio is the Reynolds number which depends on the radii and rotation rates of the cylinders and on the viscosity. Since both cylinders are allowed to rotate, we have two Reynolds numbers. If the rotation is sufficiently fast, the homogeneous fluid motion becomes unstable against a roll structure with wave vector parallel to the axis of rotation of the cylinders. This bifurcation to the so called Taylor vortices is a stationary one when the cylinders co-rotate, but when the cylinders counter-rotate the bifurcation can be oscillatory (Fig. 11). Often the patterns which occur are cylindrically symmetric, so in this 
case the anisotropy of the system leads to a description in terms of a onedimensional amplitude equation.

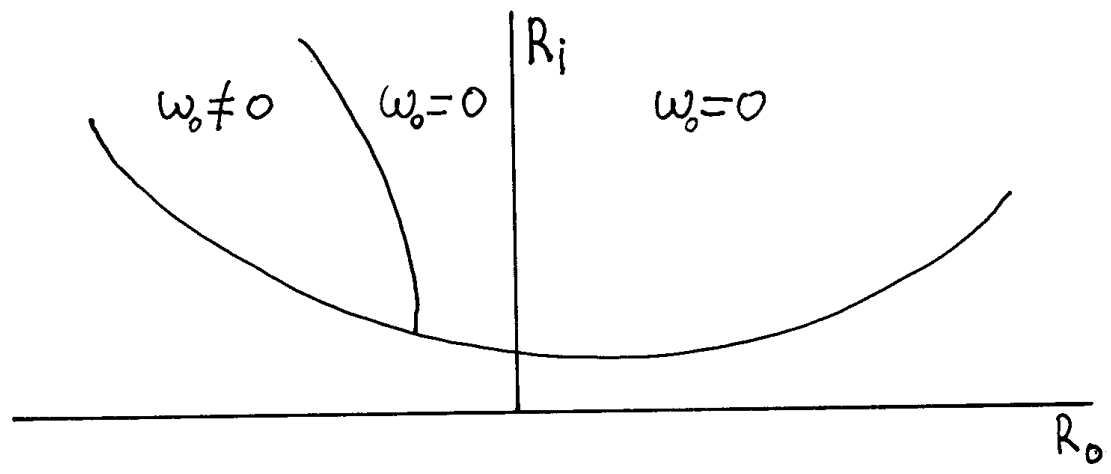

Figure 11: Stationary $\left(\omega_{0}=0\right)$ and Hopf $\left(\omega_{0} \neq 0\right)$ bifurcations for the Taylor-Couette experiment. $R_{o}$ is the Reynolds number $\Omega_{o} r_{o}\left(r_{o}-r_{i}\right) / \nu$ of the outer cylinder of radius $r_{o}, R_{i}$ is the Reynolds number $\Omega_{i} r_{i}\left(r_{o}-r_{i}\right) / \nu$ of the inner cylinder with radius $r_{i}$, and $\Omega_{o}$ and $\Omega_{i}$ are the rotation rates of the two cylinders.

\subsection{Parametric surface waves.}

If we oscillate a shallow layer of fluid in the vertical direction, this results in a modulation of gravity. For large enough modulations, the surface starts to develop waves of half the driving frequency. Because the instability occurs via a modulation of one of the parameters of the system it is called a parametric instability. From an experimental point of view, this system has the advantage that one can tune two parameters, the driving frequency, which sets length and time scales for the waves, and the driving amplitude. However, because dissipation is rather small in these systems, the patterns can be very complicated and transients die out slowly.

\subsection{Directional solidification.}

The regularity of the shapes produced by crystals growing into a supercooled or supersaturated environment is a well known phenomenon. The beautiful 
feathery patterns with the hexagonal symmetry of ice, photographed in selected snowflakes give an example of the possible richness of crystal growth. The tendency towards pattern formation in solidification is demonstrated by the instabiliy of a plane front of the solid phase growing in the supercooled liquid. This instability, known as the Mullins-Sekerka instability, can be understood as follows. When the liquid freezes, it has to transport a certain amount of heat, the so-called latent heat, into the supercooled liquid. The larger the temperature gradient at the solid-liquid interface, the faster the latent heat is conducted away and iso-temperature lines are squeezed (Fig. 12). So around a bulge in the interface the solid phase can grow faster and the bulge therefore grows. Small fluctuations of the surface will thus also be unstable. If this were the only mechanism, it would mean that perturbations

(a)

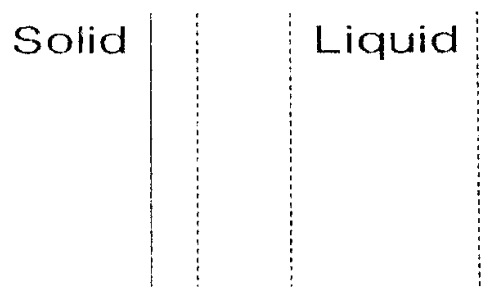

(b)

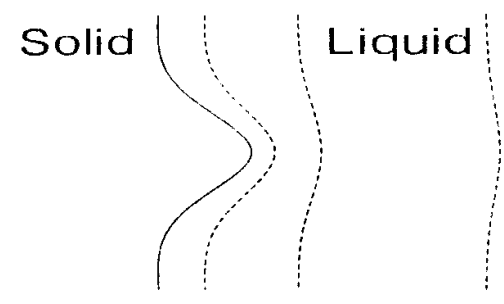

Figure 12: Sketch of the diffusion mediated instability occuring for growth of a crystal into an undercooled melt. In front of a bulge, the isotherms are squeezed, leading to better conduction of the latent heat away from the interface, and as a result to enhanced growth. For directional solidification the diffusion field associated with impurities accumulating in front of the interface gives rise to a similar instability. a) Flat interface. b) Effect on isotherms of distortion of the interface.

on all length scales would grow, leading to irregular, fractal like patterns as in diffusion limited aggregation. However, due to surface tension very steep interfaces will be damped. In the case of free growth of an interface into a supercooled region the instability does not saturate at small amplitudes, so that an analysis based on the Mullins-Sekerka instability is only qualitatively useful, for example in identifying characteristic length scales. 
In Fig. 1 we showed an example of a directional solidification pattern in a liquid crystal phase growing with constant velocity in an applied external temperature gradient. Note that when the system is just above threshold the pattern is very close to being sinusoidal in that case. However, when we move away from threshold, the amplitude grows, nonlinearities become more important and the sinus is deformed by higher harmonics. Eventually, in the last picture we see that the discrete translational symmetry parallel to the interface is broken, and that little drops of the melt are isolated in the solid, indicating strongly nonlinear behaviour. In liquid crystals the bifurcation is supercritical and the weakly nonlinear behaviour close to threshold can be described by an amplitude equation.

\subsection{Thermal noise.}

As we mentioned in section 2.5, thermal fluctuations may play a role near onset. In this section we shall list some results for the strength of the noise in various physical examples. We first consider the Rayleigh-Bénard system near threshold [6], for which the strength of the thermal noise is estimated as follows: At equilibrium, Landau and Lifshitz [7] have shown that noise can be taken into account by adding to the hydrodynamic equations (37) and (38) Langevin forces whose spectrum is white and whose strength is determined by a detailed balance condition. We furthermore assume that the noise does not change away from equilibrium, since its origin is in the molecular degrees of freedom which should be unaffected by macroscopic forcing. It is then straightforward to show $[6,8,9]$ that in a large system one is led near threshold to the stochastic amplitude equation

$$
\tau_{0} \partial_{t} A=\varepsilon A+\xi_{0}^{2}\left(\partial_{x}-\left(i / 2 q_{0}\right) \partial_{y}^{2}\right)^{2} A-g_{0}|A|^{2} A+f_{A}(x, y, t),
$$

where $f_{A}$ is a white noise source whith correlations

$$
<f_{A}(x, y, t) f_{A}\left(x^{\prime}, y^{\prime}, t^{\prime}\right)>=2 F_{A} \xi_{0}^{2} \tau_{0} \delta\left(x-x^{\prime}\right) \delta\left(y-y^{\prime}\right) \delta\left(t-t^{\prime}\right) .
$$

We will write the dimensionless noise strength in the form

$$
F_{A}=Q_{0} Q_{1}(\sigma)
$$

where

$$
Q_{0}=\frac{k_{B} T}{\rho d \nu^{2}}
$$


is the basic small parameter measuring the ratio of the thermal energy $k_{B} T$ to the characteristic dissipative energy of convection $\left(\rho d^{3}\right)(\nu / d)^{2}$. For a layer of water of depth $1 \mathrm{~cm}$. we have $Q_{0} \approx 10^{-9}$. The quantity $Q_{1}$ depends on parameters of the system (here on the Prandtl number $\sigma$ ), and can be shown [9] to be

$$
Q_{1}=\sigma d^{4} / \xi_{0}^{2} \tau_{0} \kappa \sim \sigma^{2} /(\sigma+\text { const. }) .
$$

For the Taylor-Couette system the ratio of energy scales $Q_{0}$ is equal to $k_{B} T / 2 \pi r_{o} \rho \nu^{2}$, whereas $Q_{1}$ is a function depending on the radius ratio of the outer and inner cylinders $r_{o} / r_{i}$ and on the Reynolds number $R_{o}$ of the outer cylinder [10].

For binary fluids the ratio of energies $Q_{0}$ is the same as in the RayleighBénard case, but $Q_{1}$ is a function of the Lewis number, the separation ratio and the Prandtl number [11].

In most cases the dimensionless noise strength is extremely small and effects of noise are thus difficult to study experimentally. One system where noise is enhanced is convection in gases (since $\nu$ is smaller), and another one is electrohydrodynamic convection in nematics, since very thin layers can be used. The corresponding amplitude equation is the anisotropic one (34), with a noise term of the form (46) and (47) with

$$
Q_{0}=\frac{k_{B} T}{K_{\|} d}
$$

$K_{\|}$being an orientational elastic constant. The quantity $Q_{1}$ has been recently calculated by Treiber and Kramer [12], and it depends on Prandtlnumber-like parameters representing ratios of time scales for relaxation of the momentum, the charge and the nematic director.

\subsection{Noise sustained structures.}

Even when the noise is very small, its effects can be important in systems which are convectively unstable. Let us consider the one-dimensional stochastic amplitude equation

$$
\tau_{0}\left(\partial_{t} A+s_{0} \partial_{x} A\right)=\varepsilon_{0} A+\xi_{0}^{2} \partial_{x}^{2} A-g_{0}|A|^{2} A+f_{A},
$$


in a half-space $x \geq 0$, with constant control parameter $\varepsilon_{0}>0$, group velocity $s_{0}$ and the boundary condition $A(x=0)=0$. Such a model describes for example Taylor-Couette flow with axial throughflow $[13,10]$.

Any fluctuation in the system will grow since $\varepsilon_{0}>0$, and will be advected away so long as the state $A=0$ is convectively unstable $\left(0<\varepsilon_{0}<s_{0}^{2} \tau_{0}^{2} / 4 \xi_{0}^{2}\right)$. Without noise the unstable $A=0$ state will be maintained, but if there is a constant source of noise, as in (51), then fluctuations are continually created and allowed to grow as they advect downstream. At some distance $x_{0}$, which depends on the noise strength $F_{A}$ and on $s_{0}$, the order parameter grows to macroscopic size and eventually saturates to its bulk value $\left|A_{0}\right|^{2}=$ $\varepsilon_{0} / g_{0}$. Thus a measurement of $x_{0}$ can yield information on $F_{A}$, if the other parameters in (51) are known. Note that it is essential that translational invariance is broken in the system, otherwise the advection term $s_{0} \partial_{x} A$ could be transformed away by a change of reference frame.

In a recent experiment on Taylor-Couette flow with througflow, Babcock et al. [13] were able to fit their data to a complex generalisation of Eq. (51), and to determine the small noise strength $F_{A} \approx 5 \times 10^{-9}$, which is however larger than the estimate for thermal noise [10] by a factor of roughly 200 . The origin of this discrepancy is at present unknown.

Recently, Schöpf and Rehberg have performed measurements of the thermal noise strength in a binary fluid convection experiment in the convectively unstable regime [14]. In this case, the measured and theoretically predicted strengths of the thermal noise are in very good agreement. 


\section{Beyond the phase winding solutions.}

In the previous sections, we have derived the amplitude equations and indicated their validity for a number of physical systems. These equations provide us with a proper starting point for answering some of the questions we posed in section 1.2 in the context of Rayleigh-Bénard convection. Some specific theoretical issues are:

- Which (near) periodic patterns out of a continuum of possibilities naturally emerge above threshold, i.e. what is the mechanism of "pattern selection"? Initial conditions and boundary conditions play an important role in this. This question arises especially for the RGL equation and for the CGL equation with $\left|c_{1}\right|,\left|c_{3}\right| \ll 1$.

- To what extent is the long-time dynamics dominated by structures like defects or other elementary objects (often refered to as coherent structures - see below).

- In addition, for the CGL equation with the Newell criterion (32) satisfied, chaotic solutions are expected. What are the proper quantities to characterize these solutions in large systems where many degrees of freedom come into play ${ }^{10}$ ?.

These questions are at the heart of modern research on nonequilibrium pattern formation. From a theoretical point of view one may start by attempting to understand various types of solutions of the amplitude equations, their stability, and their basins of attraction (i.e. the set of initial conditions from which they are reached).

Providing a comprehensive overview of our present understanding of these issues is far beyond the scope of this chapter. We will therefore content ourselves in this last section with giving some flavor of recent work on these problems by showing some examples. The reader interested in a more thorough discussion should consult the relevant sections of the review by Cross and Hohenberg cited in sec. 6 and the references therein.

We shall start by showing a simulation of the evolution of a two-dimensional pattern from an Eckhaus unstable to a stable state in the RGL equation. This

\footnotetext{
${ }^{10}$ Actually, this question may also be relevant in regimes where $c_{1} c_{3}<1$, as studied in [15], in which spatio-temporal chaos also arises.
} 
naturally leads to the subject of defects. We then briefly show some of the new phenomena which arise in the complex equation. This will lead to the introduction of coherent structures in the one-dimensional amplitude equation, structures which are closely related to the defects mentioned above.

\subsection{The Eckhaus instability}

The two-dimensional RGL equation (35) for anisotropic systems with $\xi_{0}$ and $g_{0}$ scaled out as in equation (27), admits plane wave solutions of the form $A=a e^{i q x}$ with wavevector parallel to the $x$-axis. These waves exist for $q^{2}<\varepsilon$ and are Eckhaus stable if $q^{2}<\varepsilon / 3$. If we prepare the system in a plane wave state with $\varepsilon / 3<q^{2}<\varepsilon$, it will be Eckhaus unstable, and we might expect it to evolve into a state inside the Eckhaus stable band. This is indeed what happens as illustrated in Fig. 13, which shows a simulation of the two-dimensional RGL equation. In large parts of the pattern, the variations of the modulus of the amplitude are relatively small, so the pattern can be described by the phase $\phi$ of $A$, which is defined by writing $A=a e^{i \phi}$. For a phase winding solution of the RGL equation we have $\phi=q x, a=$ constant. The phase variable $\phi$ occurs naturally when one looks at nearly periodic patterns. For instance, in the picture of a Rayleigh-Bénard experiment in Fig. 3, the phase of the signal was used to depict the pattern. In Fig. 13 the black curves indicate regions where the phase is 0 , and the white curves regions where the phase is $\pi$, say.
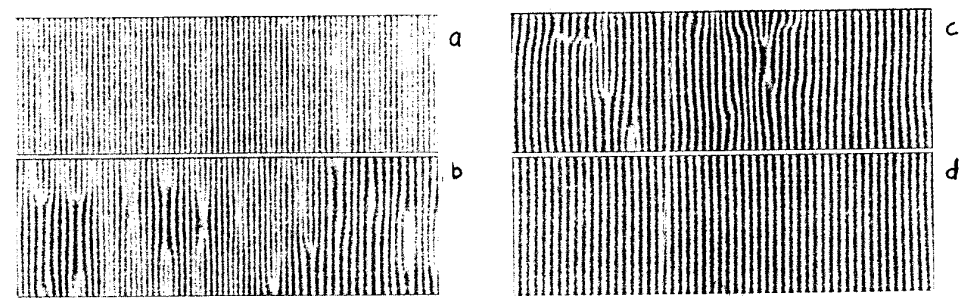

Figure 13: Four subsequent stages in the simulation of the two-dimensional amplitude equation (35), evolving from an Eckhaus unstable to an Eckhaus stable phase winding solution. After [16].

The simulations we show were done with the RGL equation with $\varepsilon$ scaled 
out, which amounts to setting $\varepsilon=1$. The system was prepared in an Eckhaus unstable initial state with $q=0.7$. In panel (a) we see a weakly modulated periodic pattern. These modulations grow in strength, and in panel (b) we see that some of the equal phase curves merge, reducing the average wave number. Where such curves merge, the phase becomes singular, and if $A$ is smooth it has to go through zero. This is analogous to our previous discussion of phase-slips in section 2.3. In this two-dimensional case the singularities in the phase are associated with topological defects, and they play an important role in the selection of final states of patterns. In panel (c) many of the defects have annihilated and the pattern is close to a periodic Eckhaus stable state with lower wave number, which is finally reached in panel $(d)$. For a more thorough discussion of the Eckhaus instability we refer the reader to $(\mathrm{CH} I V$ A 1 a ii).

\subsection{Topological defects}

The previous discussion on the Eckhaus instability introduced defects in a somewhat sketchy way. We sharpen our formulation by considering a twodimensional pattern in which over some large closed loop $C$ the phase $\phi$ varies slowly, but for which the integer valued integral

$$
(1 / 2 \pi) \oint_{C} \vec{\nabla} \phi \cdot \overrightarrow{d l}
$$

is equal to 1 . It is not difficult to see that this is not consistent with a slow variation of the phase everywhere: If we smoothly shrink the contour, the integer value of the integral remains smooth if the phase is smooth. The integral is therefore a nonzero constant for arbitrarily small loops, which clearly contradicts the assumption of smoothness of $\phi$. Thus a non-zero value of an integral of the form (52) necessarily implies the existence of at least one topological point defect inside the loop, where the assumption of slow variation breaks down. Such a defect is called topological, because a smooth deformation of the phase field does not influence its existence. The motion of a defect depends both on the pattern in its neighbourhood and on the long-range interaction with other defects $(\mathrm{CH} \mathrm{V} \mathrm{B)}$.

The notion of a topological defect is related to broken symmetries (CH IV B 2). As an example we may look at the onset of convection in a RayleighBénard experiment. Below threshold the system is uniform and arbitrary 
translations do not change the solution, so they are all symmetry transformations. Above threshold when a periodic pattern has emerged, arbitrary translations are no longer symmetries of the system. If we apply a translation to a periodic pattern we obtain in general a different, but entirely equivalent, pattern. The different patterns that can be generated by applying all continuous symmetries of the system below threshold, in this case all translations, can be labelled by phase variables. In the case of a periodic pattern this is simply an element of $\mathrm{SO}(2)$. A spatially uniform change of any of the phase variables produces a new solution and therefore no dynamics. A slowly varying spatial change of the phase variables is expected to relax slowly in time, and therefore phase variables can be used to describe the dynamics of slow spatial variations of the basic pattern. This remains true further above threshold as well. We will not go into this subject in more detail, but refer the reader to ( $\mathrm{CH}$ IV A 2).

\subsection{The Benjamin-Feir instability}

The local structures discussed above have a topological origin, but there also exist local structures which do not have topological significance. In particular, as illustrated below, coherent structures in the one-dimensional amplitude equation show features reminiscent of defects in two dimensions when viewed in a two-dimensional space-time plot (compare Fig. 14c). However, there is not necessarily a topological charge associated with such structures, even though both types of solutions have similar behaviour.

In many situations local structures seem to be long lived, and are therefore important for the selection of final states, as was illustrated by the defects in the simulation of the Eckhaus instability of the RGL equation in Fig. 13.

The stability diagram for phase winding solutions of the CGL equation is very similar to the one for the RGL equation (Fig. 7a) as long as $\left|c_{1}\right|,\left|c_{3}\right| \ll 1$. In this parameter range the dynamics following the initial state of Fig. 13 would qualitatively resemble that of the RGL, except for the fact that the near-periodic pattern now corresponds to traveling waves. As we saw in section 2.3, however, when the product $c_{1} c_{3}$ increases the band of stable phase winding solutions shrinks, and if we satisfy the Newell criterion $c_{1} c_{3}>1$, there are no stable phase winding solutions of the form $A=a e^{-i \omega t+i q x}$ left. 


\subsubsection{Spatio-temporal chaos.}

Figures $14 \mathrm{a}$ and $14 \mathrm{~b}$ in which the amplitude $|A|$ is plotted vs. $x$ at regular time intervals, illustrate the chaotic behaviour typically observed when $c_{1} c_{3}>$ 1. From these figures we see that in the limit where both $c_{1} c_{3} \gg 1$ and $c_{3} \gg 1$, intermittent structures that we will refer to as "pulses" are found. In Fig. $14 \mathrm{c}$, a grey-scale space-time plot of the phase $\phi$ is shown for a simulation with $c_{1}=2, c_{3}=1$. The simulation started with random initial conditions, but the long-time dynamics illustrated here is qualitatitvely independent of the initial conditions. In this figure, lines where $\phi=0$ are white and lines where $\phi=\pi$ are black, say. Moving up along the vertical time axis shows the behavior of the phase $\phi$ at a fixed position $x$ as a function of time. The defect-like points where two black or two white curves merge correspond to phase slip events in space-time.

This chaotic behaviour brings up a number of new questions. The understanding of chaotic systems with only a few degrees of freedom, for which the complex behaviour occurs in the time evolution only, has greatly increased the past 20 years. The situation in spatially extended systems on the other hand, such as the CGL equation, which have a large or even infinite number of degrees of freedom, is much more complicated and far less well understood. Since the state at a fixed time can be very complex, this behaviour is often referred to as spatiotemporal chaos.

It has been suggested that the limit of an infinite system, i.e. extensive chaos, might be described using concepts borrowed from statistical mechan-

ics and critical phenomena. In particular, the chaotic behaviour shown in Fig 14c has been analyzed in terms of correlation functions of the phase [15], but studies along these lines have only just begun. It has recently been suggested that some phase correlation functions in these chaotic regimes might be related to the behaviour of interface growth models [17]. We also note that some experiments on Rayleigh-Bénard convection in binary mixtures in an annulus [18] show chaotic behaviour reminiscent of that in the left two pictures of Fig 14, but a detailed comparison between simulations and experiments has not been made. 

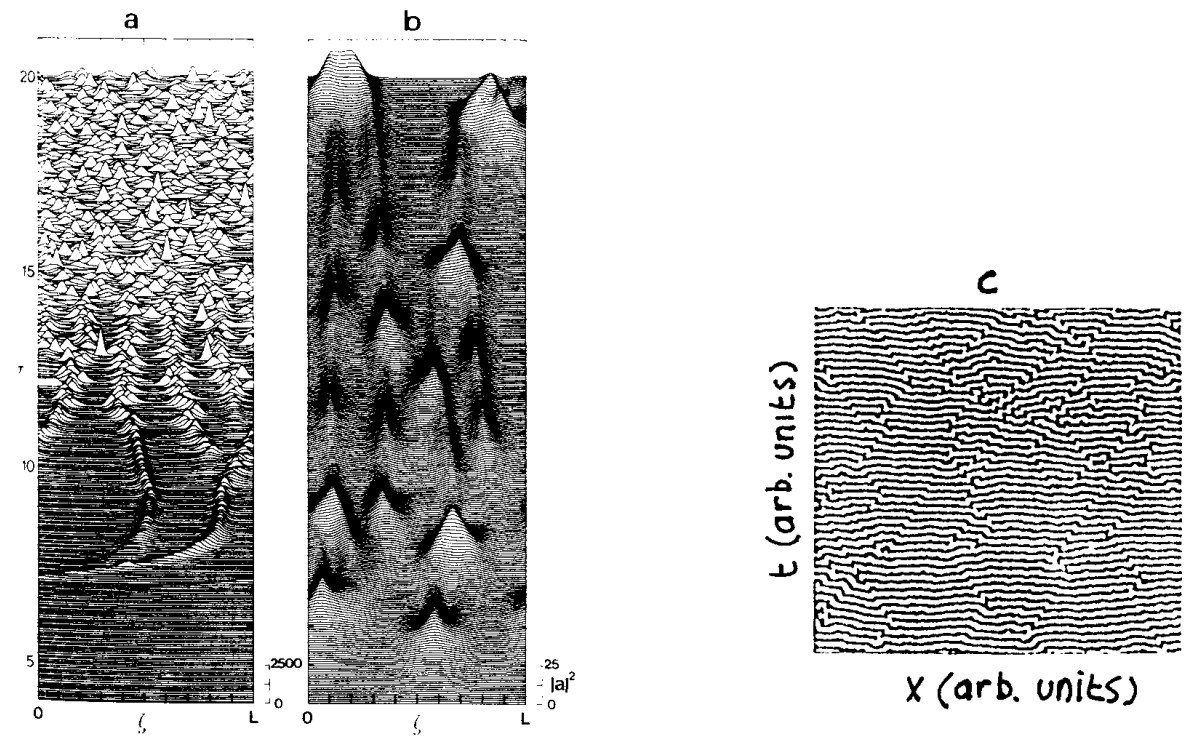

Figure 14: Time evolution of a chaotic state of the one-dimensional CGL equation. (a),(b) Simulations of the amplitude equation for a case with $c_{3} \rightarrow \infty$ (after rescaling, the limit $c_{3} \rightarrow \infty$ amounts to setting the real part of the prefactor of the cubic term in the CGL equation equal to zero, so that it reads $i|A|^{2} A$ ) and $c_{1}=100$ in (a) and $c_{1}=1$ in (b); the initial condition was white noise with spatial average $\overline{|A|^{2}}=.01$. After [19]. (c) Grey scale plot of the phase in a simulation of the CGL equation with $c_{1}=2$ and $c_{3}=1$, which started with random initial conditions. After [15] .

\subsection{One-dimensional coherent structures.}

As Fig. 14 illustrates, in the region $c_{1} c_{3}>1$ the dynamics of the CGL equation displays spatiotemporal chaos. In other regions of parameter space, one often finds that part of the dynamics is governed by well-defined regular solutions which we will refer to as coherent structures (some of them also exist in the RGL equation). Coherent structures are solutions that are either themselves localized or that consist of domains of regular patterns connected by localized defects or interfaces. It is important to understand the existence and stability of these coherent structures as a first step towards an analysis of the dynamics of more complicated patterns. We shall now give a brief 
overview of what has been learned in the past few years about the four coherent structures of the one-dimensional CGL equation shown in Fig. 15. All the solutions we shall discuss are uniformly translating, i.e. are solutions of the CGL equation (29) of the form

$$
A(x, t)=e^{-i \omega t} \hat{A}(x-v t) .
$$
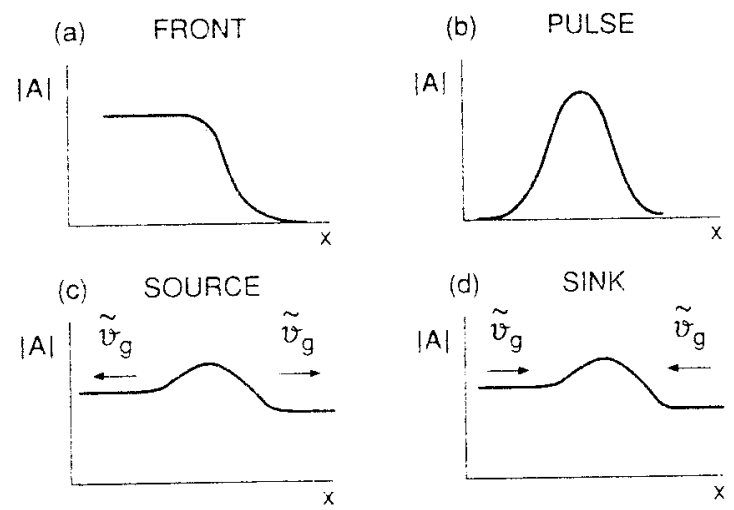

Figure 15: Schematic representation of coherent structures of the onedimensional complex amplitude equation.

\subsubsection{Sources and sinks.}

Let us first consider solutions which connect one phase winding solution on the left to another one on the right. We will call them domain wall or shock type solutions. In a space-time plot like that of Fig. 14c, these solutions would appear like "grain boundaries" between domains of different wave number. Since a traveling wave has a nonzero group velocity $v_{g r}$, there are several possibilities depending on whether the group velocity of each phase winding solution points away from or towards the localized structure connecting the two asymptotic states. It is useful to use the group velocity $\tilde{v}_{g r}$ relative to the velocity $v$ of the localized structure $\tilde{v}_{g r} \equiv v_{g r}-v$. Thus $\tilde{v}_{g r}$ is the velocity of a small perturbation of a phase winding solution in the frame moving with the domain wall. If the relative group velocity points away from 
the localized structure on both sides, the latter has the properties of a source, while if $\tilde{v}_{g r}$ points inwards on both sides, we will call the domain wall a sink $[20],[21]$. In principle, solutions with the relative group velocities pointing in the same direction on both sides of the wall might also be possible, but one can show [21] that such solutions do not exist in the cubic CGL equation. They do, however, exist in higher-order extensions, such as the quintic CGL equation mentioned below. In the RGL equation, most of the dynamics of domain walls can be understood in terms of the tendency of walls to move in the direction of lower "free energy" [see the discussion in 2.2]. The behavior of sinks and sources in the CGL equation is more complicated.

Sinks are in a sense rather dull objects, since the very fact that the relative group velocities point inwards means that the phase winding solutions that are connected must originate in some other regions of space. One then tends to focus on the dynamics in these regions. Nevertheless, the velocity of sinks is important during transients because it determines which regions shrink and which ones expand. In the one-dimensional CGL equation, there is typically a two-parameter family of sinks for not too large velocities [21]. This means that if we select two arbitrary asymptotic phase winding solutions, one on the left and one on the right, with group velocities pointing inwards, there is always a sink solution with some uniquely determined velocity $v$ that connects these two states. The stability of sinks has to our knowledge not been studied in detail, although one expects them to be stable over a range of parameters.

Sources send out waves, and so may determine the large-time asymptotic dynamics. On the basis of an analysis of the ordinary differential equations that can be derived for the function $\hat{A}$ in Eq. (53) one generically expects the existence of a $v=0$ source, as well as a discrete set of $v \neq 0$ sources [21]. In other words, one expects that in addition to the $v=0$ solutions there are only sources with particular values of the velocity and asymptotic wave numbers. Surprisingly, however, Bekki and Nozaki [22] found a continuous family of exact source solutions of the CGL equation. The existence of a family rather than a discrete set of sources was interpreted by van Saarloos and Hohenberg [21] as a hint that there might be some hidden symmetry or some accidental nongenericity in the cubic CGL equation for all parameter values. Support for this point of view comes from recent work by Popp em et al. [23], who discovered that if a small perturbation is added to the CGL equation, the stability of these solutions depends sensitively on the sign and strength of the perturbation. For certain ranges of the parameters $c_{1}$ 
and $c_{3}$, the Bekki-Nozaki source solutions seem to play an important role in the chaotic dynamics of the CGL equation [23]. Recently, Lega et al. [24] observed holes reminiscent of the Bekki-Nozaki type solutions in a low Prandtl number convection experiment.

\subsubsection{Pulses}

The work on pulse type solutions of the type sketched in Fig. 15b was motivated largely by observations of localized convective regions in experiments on binary mixtures (CH IX A 2). Since the instability to traveling waves in this system actually corresponds to a subcritical bifurcation (compare section 1.4.1), this motivated a number of groups to study the following quintic extension of the CGL

$$
\frac{\partial A}{\partial t}=\left(1+i c_{1}\right) \frac{\partial^{2} A}{\partial x^{2}}+\varepsilon A+\left(1+i c_{3}\right)|A|^{2} A-\left(1-i c_{5}\right)|A|^{4} A
$$

Note that the real part of the prefactor of the cubic term is now positive, so that this term is destabilizing instead of stabilizing. The quintic term now plays the stabilizing role, giving rise to the subcritical behaviour sketched in Fig. 5. Perturbation expansions about both the relaxational limit $\left(c_{i} \rightarrow 0\right)$ and the Hamiltonian limit $\left(c_{i} \rightarrow \infty\right)$ [21] have shown that there exist stable pulse solutions with zero velocity in large subcritical $(\varepsilon<0)$ ranges of the $\varepsilon, c_{1}, c_{3}, c_{5}$ parameter space. Pulse solutions can become unstable by splitting into two fronts which move apart. The long time properties of each front are then given by that of a single front like the one shown in Fig. 15a, so one can get information on the range of existence of pulses by analyzing the dynamics of a single front. Pulse solutions of (54) are stationary in the frame moving with the group velocity of the traveling waves; in principle, if one considers an amplitude expansion near a weakly subcritical bifurcation (meaning that the prefactor of the real part of the destabilizing cubic term is small), nonlinear gradient terms will arise in the same order as the quintic term $|A|^{4} A$, thus leading to a drift velocity for pulses slightly different from the group velocity. Experimentally, however, pulses are found to have a drift velocity much smaller than the group velocity [25]. Although this can be accounted for on an ad-hoc basis by adjusting the coefficient of the nonlinear gradient term to be large, a more fundamental analysis [26] attributes the small drift velocity of pulses to the coupling of the amplitude $A$ to the slow 
concentration field. This represents a correction to the amplitude expansion due to the existence of an extra slow variable.

\subsubsection{Fronts}

The dynamics of fronts in the cubic CGL equation for $\varepsilon>0$ is relatively well understood in terms of so-called marginal stability selection criteria (see [21] and references therein). Because of its importance for the stability of pulse solutions, front propagation has recently been studied in great detail[21] in the quintic equation (54) for both $\varepsilon>0$ and $\varepsilon<0$. It turns out that an exact nonlinear front solution can be found, whose dynamics plays an important role in the selection of patterns. Together with a set of rules and conjectures a fairly complete picture of the stability of pulses and of the dynamics of fronts has emerged. Some of the surprising findings are:

(i) In some ranges of the parameters, pulses can remain stable in the limit $\varepsilon \uparrow 0$. In these regions, fronts only advance into the state $A=0$ for $\varepsilon>0$, and dynamically the distinction between the supercritical and subcritical cases seems to have blurred.

(ii) For uniformly translating fronts to propagate into the $A=0$ state, the net nonlinear dispersion has to be relatively small, i.e. $c_{3} \approx-c_{5}$.

(iii) It is possible to have fronts whose edge propagates with a well-defined

velocity $v^{*}=2 \sqrt{\varepsilon\left(1+c_{1}^{2}\right)}$ for $\varepsilon>0$, that are not uniformly translating, i.e. not of the form (53).

(iv) There are subcritical regions of parameter space where chaotic pulses spread; these are sometimes refered to as "slugs".

$(v)$ In the limit where the $c_{i} \rightarrow \infty$ there are dynamically important front solutions which cannot be obtained perturbatively from the Hamiltonian limit $c_{i}=\infty$.

\section{Concluding remarks.}

In the bulk of this chapter we have focused primarily on the derivation of amplitude equations for pattern forming systems. The amplitude equation approach brings out the common features of patterns in different systems near threshold. Even if the starting equations describing a particular system are not well known or too complicated, one expects on general grounds that 
near the threshold of a finite wave number instability (which itself could be a secondary instability), the amplitude equation will capture the essential behaviour both qualitatively and quantitatively. Of course, the reader should keep in mind that the behaviour away from threshold is much richer than that predicted by amplitude equations, as illustrated by the Busse balloon of Fig. 10 for the case of the Rayleigh-Bénard instability.

\section{Suggested further reading}

- M. C. Cross and P. C. Hohenberg, "Pattern formation outside of equilibrium", Rev. Mod. Phys. 65, 851 (1993). For a shorter introduction, see P. C. Hohenberg and M. C. Cross, "An introduction to pattern formation in nonequilibrium systems", in Fluctuations and Stochastic Phenomena in Condensed Matter (Springer Verlag, NY), 55 (1987).

- A. C. Newell, T. Passot and J. Lega, "Order parameter equations for patterns", Ann. Rev. Fluid Mech. 25, 399 (1993).

\section{References}

[1] J. Bechhoefer, A. J. Simon, A. Libchaber and P. Oswald, Phys. Rev. A40, 2042 (1989).

[2] D. Bensimon, B. I. Shraiman and V. Croquette, Phys. Rev. A38, 5461 (1988).

[3] V. Croquette, M. Mory and E. Schosseler, J. Phys. (Paris) 44, 293 (1983).

[4] S. Chandrasekhar, Hydrodynamic and Hydromagnetic Stability, Clarendon Press, Oxford (1961).

[5] F. H. Busse, Rep. Prog. Phys 41, 1929 (1978); F. H. Busse and R. M. Clever, J. Fluid Mech. 91, 319 (1979).

[6] J. B. Swift and P. C. Hohenberg, Phys. Rev. A15, 319 (1977). 
[7] L. D. Landau and E. M. Lifshitz, Fluid Mechanics, Addison-Wesley, Reading, MA (1959) Chap. XVII.

[8] R. Graham, Phys. Rev. A10, 1762 (1974); Erratum Phys. Rev. A45, 4198 (1992).

[9] P. C. Hohenberg and J. B. Swift, Phys. Rev. A46, 4773 (1992).

[10] J. B. Swift, K. L. Babcock and P. C. Hohenberg, Physica A (to be published).

[11] W. Schöpf and W. Zimmerman, Phys. Rev. E47, 1739 (1993).

[12] M. Treiber and L. Kramer, Phys. Rev. E (to be published).

[13] K. Babcock, G. Ahlers and D. S. Cannell, Phys. Rev. Lett. 67, 3388 (1991).

[14] W. Schöpf and I. Rehberg, submitted to J. Fluid Mech.

[15] B. I. Shraiman, A Pumir, W van Saarloos, P. C. Hohenberg, H. Chaté and M. Holen, Physica D57, 241 (1992).

[16] A. Weber, E. Bodenschatz and L. Kramer, Adv. Mater. 3, 191 (1991).

[17] G. Grinstein, D. Mukamel, R. Seidin and C.H. Bennett, Phys. Rev. Lett. 70, 3607 (1993).

[18] J. A. Glazier, P. Kolodner and H. Williams, J. Stat. Phys. 64, 945 (1991).

[19] C. S. Bretherton and E. A. Spiegel, Phys. Lett. A96, 152 (1983).

[20] See e.g. P. Coullet, T. Frisch and F. Plaza, Physica D62, 75 (1993) and references therein.

[21] W. van Saarloos and P. C. Hohenberg, Physica D56, 303 (1992).

[22] N. Bekki and N. Nozaki, Phys. Lett. A110, 133 (1985).

[23] S. Popp, O. Stiller, I. Aranson, A. Weber and L. Kramer, Phys. Rev. Lett. 70, 3880 (1993). 
[24] J. Lega, B. Janiaud, S. Jucquois and V. Croquette, Phys. Rev. A45, 5596 (1992).

[25] P. Kolodner, Phys. Rev. Lett. 66, 1165 (1991).

[26] H. Riecke, Phys. Rev. Lett. 68, 301 (1992). 